\title{
Vibrations due to Flow-Driven Repeated Impacts
}

\author{
Sumin Jeong and Natalie Baddour \\ Department of Mechanical Engineering, University of Ottawa, 161 Louis Pasteur, Ottawa, ON, Canada K1N 6N5
}

Correspondence should be addressed to Natalie Baddour; nbaddour@uottawa.ca

Received 15 March 2013; Accepted 21 May 2013

Academic Editor: Jia-Jang Wu

Copyright (C) 2013 S. Jeong and N. Baddour. This is an open access article distributed under the Creative Commons Attribution License, which permits unrestricted use, distribution, and reproduction in any medium, provided the original work is properly cited.

\begin{abstract}
We consider a two-degree-of-freedom model where the focus is on analyzing the vibrations of a fixed but flexible structure that is struck repeatedly by a second object. The repetitive impacts due to the second mass are driven by a flowing fluid. Morison's equation is used to model the effect of the fluid on the properties of the structure. The model is developed based on both linearized and quadratic fluid drag forces, both of which are analyzed analytically and simulated numerically. Conservation of linear momentum and the coefficient of restitution are used to characterize the nature of the impacts between the two masses. A resonance condition of the model is analyzed with a Fourier transform. This model is proposed to explain the nature of ice-induced vibrations, without the need for a model of the ice-failure mechanism. The predictions of the model are compared to ice-induced vibrations data that are available in the open literature and found to be in good agreement. Therefore, the use of a repetitive impact model that does not require modeling the ice-failure mechanism can be used to explain some of the observed behavior of ice-induced vibrations.
\end{abstract}

\section{Introduction}

In this paper, we consider the analysis of the vibrations of a structure that is repeatedly struck by a second object. The repetitive nature of the impacts of the second, free object on the primary (assumed fixed but flexible) structure is due to a flowing fluid that repeatedly drives the free object onto the primary structure. The analysis of this model is based on two equations from mechanics: Morison's equation and the conservation of momentum. Morison's equation is adopted to add the influence of fluid flow on the properties of the structure that is assumed to be immersed in the fluid. The conservation of momentum is combined with the coefficient of restitution to derive the model in which the force on the structure is considered to be a series of impacts due to the free object being driven by the fluid flow. Numerical simulations are conducted to obtain insight into the dynamics predicted by the model. This model is proposed and investigated in order to attempt to explain some of the observed nature of ice-induced vibrations.

\section{Motivation}

Research analyzing ice mechanics has been conducted by the National Research Council of Canada, the Canadian
Coastguard, U.S. Navy, various universities, the offshore industry, and several oil companies [1]. In the early stages, field and laboratory experiments were used to investigate the mechanism for ice-induced vibrations (IIV). Blenkarn [2] presented ice force data which he recorded in drilling platforms of Cook Inlet from the winter of 1963 to 1969. His paper became the basis of the negative damping or selfexcited model. Toyama et al. [3] suggested phase division of an ice forcing function based on small scale tests and this approach was also used by other researchers. Ranta and Räty [4] also proposed a similar idea of phase division. A more recent experimental publication was presented by Barker et al. [5], in which extensive small scale tests of wind turbines in Danish waters were investigated. The results provided information on ice forces which vary with the shapes of structures. Recent publications of Sodhi's experiments [6-8] also contributed to the understanding of the dependency of ice forces on ice velocities.

Along with the experimental progress, theoretical models of IIVs were also proposed by attempting to define the origin of the vibrations. After Peyton's early publication [9], Neill [10] suggested that crushed ice tends to break into a certain size. He proposed that the fracture size and velocity of the accompanying ice sheet determine a characteristic failure 
frequency which in turn decides the forcing frequency [6]. This point of view defined the origin of IIVs as being via a characteristic failure mechanism, a point of view which was supported by Matlock et al. [11]. In contrast to the characteristic failure mechanism, Blenkarn [2] explained IIVs as a self-excited vibration due to negative damping. According to this theory, IIVs originate from the interaction between a flexible structure and decreasing ice crushing forces with increasing stress rate. Määttänen, one of the big contributors of the self-excited model, verified his model through field and laboratory experiments $[7,12,13]$. Similarly, $\mathrm{Xu}$ and Wang [14] also proposed an ice force oscillator model based on the self-excited model.

As research in the field progressed, different physical processes to explain the origin of the ice force were proposed. In particular, attention was turned to whether the ice was considered to fail in crushing (compression) or in bending. It is thought that compressive (crushing) failures occur as a result of ice interacting with a narrow vertical (cylindrical) structure [15]. Tsinker defines crushing as the complete failure of granularization of the solid ice sheet into particles of grains or crystal dimension; no cracking, flaking, or any other failure mode occurs during pure crushing. Specific models that correspond to ice in crushing failure are given in $[3,16]$. Even though these two articles model the same physical phenomena, they are different in their modelling approaches.

The concept of adding ice-breaking cones to cylindrical structures was proposed in the late 1970s, changing the effective shape of the structure from cylindrical to conical. The ice force on a conical structure is smaller than the force on a cylindrical structure of similar size $[5,8,17,18]$. It is thought that the main reason for the reduction in ice force is that a well-designed cone can change the ice-failure mode from crushing to bending. It is considered that the primary failure mechanism for ice interacting with a conical structure is that of bending failure. In this failure mode, the ice sheets impacting on a cone fail by bending and typically the ice breaks almost simultaneously in each event of ice failure. With conical structures and bending ice failure, analytical models are more likely to be characteristic failure frequency models that were initially proposed in the $70 \mathrm{~s}^{\prime}$, since the ice force can effectively be modelled as only depending on the properties of the ice. With conical structures, the local ice forces were experimentally found to drop to almost zero after each event of ice failure $[19,20]$ and are consequently modelled analytically with a periodic function. For example, the forcing function suggested by $\mathrm{Qu}$ et al. [21], is a simple one-degree-of-freedom model corresponding to a periodic ice force. This model is based on their own previous work $[19,20]$ as well as that of Hirayama and Obara [18]. This model attempts to represent an ice sheet failing through the bending mode and simply models the forcing function as a saw-tooth shaped, periodic force. From a modelling point of view, the bending failure of ice is easier to model since the ice-forcing function is periodic and is decoupled from the motion of the structure. This type of model thus becomes a regular forced vibration problem and once the form of the external ice force is chosen, it is a relatively straightforward problem to solve.
Despite extensive studies, no theoretical model completely explains the IIV mechanism. The variety of modelling approaches and dependence of the ice load on the structural properties have caused modelling and design difficulties. For instance, there is no method for choosing the proper dynamic ice case for the design and optimization of an ice-resistant jacket platform [22, 23]. In addition, most IIV models do not consider the influence of fluid flow even though most structures subjected to IIVs are offshore structures. Since fluid flow is one of the main driving forces of IIVs, it can contribute to the dynamics of ice forces as well as that of the structure but it has not been considered in past research.

Since the first modeling approaches were introduced, no major advances in IIV modeling have been proposed and there is no general consensus on one correct approach. All existing modeling approaches analyze the process of IIVs from a microscopic point of view, with a heavy emphasis on the mode in which the ice fails. For example, in [5] numerous small scale tests were conducted to attempt to distinguish ice failures into four different modes by the sizes of failed ice pieces and the structural responses. The procedure of defining ice-failure modes is thus neither clear nor generally agreed upon.

In this paper, we propose that a macroscopic point of view may give a different insight. For instance, a detailed analysis of all possible deformations is not generally considered when a collision of two particles is analyzed. In that case, the whole collision process can be efficiently analyzed with a single parameter, the coefficient of restitution. The main idea of this paper is that if the collision of the particles occurs repetitively, the movement of the two particles may resemble those of IIVs.

This simple concept provides the inspiration for the new modeling approach proposed in this paper. The vibration characteristics of IIVs are proposed as being the result of a repetitive forcing function, with the moving fluid repeatedly pushing the ice towards the structure. If all the microscopic ice-failure processes can be condensed into a single macroscopic parameter, the coefficient of restitution, the remaining vibration characteristics are similar to those of repetitive, driven collisions of two particles. The ice is modeled as one of the particles in the collision and the structure is the other. IIVs can then be modeled and simulated by using the conservation of momentum along with the coefficient of restitution, which is a completely different perspective from existing IIV models that take a microscopic view and focus on the precise mechanism of ice failure.

\section{Modeling of Flow-Driven Repeated Impacts with the Conservation of Momentum}

We consider a two degree-of-freedom system that consists of two masses. Figure 1 is a schematic diagram of two masses which collide repetitively. Mass $M$ represents the free mass and mass $m$ represents the flexible structure.

The force which drives the free mass needs to be modeled so that collisions occur repetitively. The free mass is driven by various external forces such as wind and thermal expansion, 


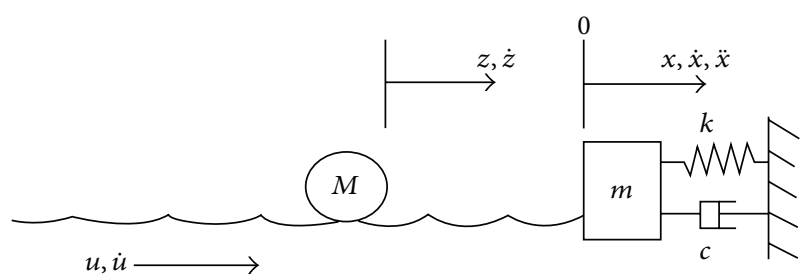

Figure 1: Mechanical model of the system.

but the main driving force is fluid flow. Depending on the initial displacement and velocity of the flow, the velocity of the free mass is determined just prior to the time of impact, and the maximum velocity of the free mass is taken to be the velocity of the flow. The free mass moves until it collides with the structure. After the impact, the free mass either stops, moves forward, or bounces back in the opposite direction depending on the coefficient of restitution and conservation of total system (free mass and structure) momentum. Since the free mass and fluid flow now have a relative velocity with respect to each other, the velocity of the free mass is changed by the effect of fluid drag until the free mass reaches the velocity of the flow and then moves again towards the structure for another impact. The free mass moves towards the structure and transfers its momentum to the structure again when the relative displacement between the mass and structure becomes 0 (i.e., when a collision occurs). In some cases, the structure can transfer momentum to the mass when the momentum of the structure is larger then that of the mass. This whole process keeps repeating, but due to structural damping and fluid drag, the momenta which are transferred to each other gradually decrease.

Since mass $M$ is floating in the flow, it is free to move. The flow, however, moves with its own velocity $\dot{u}$, which in this case is always assumed to be positive (to the right in the figure). The displacement of the free mass is given by the variable $z$ and that of the structure is given by the variable $x$. The mass is driven by the drag force, $f_{D}$, which is present when there is a relative velocity between the flow and the mass. There are four conditions depending on the relative velocities.

(i) When $\dot{z} \geq 0$ and $|\dot{z}| \geq|\dot{u}|, f_{D} \leq 0$.

(ii) When $\dot{z} \geq 0$ and $|\dot{z}|<|\dot{u}|, f_{D}>0$.

(iii) When $\dot{z}<0$ and $|\dot{z}| \geq|\dot{u}|, f_{D} \geq 0$.

(iv) When $\dot{z}<0$ and $|\dot{z}|<|\dot{u}|, f_{D}>0$.

Outside of a collision with the structure, no force is transmitted to the free mass except the drag force due to the fluid. The structure imparts a force to the free mass when a collision between them occurs. The drag force from the fluid is defined as

$$
f_{D}=-\frac{1}{2} \rho C_{D} A(\dot{z}-\dot{u})^{2},
$$

where $\rho, C_{D}$, and $A$ are the density of the fluid, drag coefficient, and area related to the drag coefficient, respectively [24]. The drag force which influences the system of Figure 1 changes the forcing direction according to the relative velocities, as explained above. The drag force for the free mass of Figure 1, therefore, is expressed as

$$
f_{D}=-\frac{1}{2} \rho C_{D} A|\dot{z}-\dot{u}|(\dot{z}-\dot{u}) .
$$

The equation of motion of the free mass can be obtained from (2) since no other forces are involved and is thus given as

$$
M \ddot{z}=-\frac{1}{2} \rho C_{D} A|\dot{z}-\dot{u}|(\dot{z}-\dot{u}) .
$$

Since (3) is a nonlinear equation, it should be linearized or simulated numerically. The linearized version of (3) is developed by assuming a small relative velocity. Equation (3) is linearized as

$$
M \ddot{z}=-b(\dot{z}-\dot{u}),
$$

where

$$
b=\frac{1}{2} \rho C_{D} A|\dot{z}-\dot{u}| .
$$

$|\dot{z}-\dot{u}|$ is assumed to be small and constant. The solution of (4) consists of homogeneous and particular solutions and can be obtained as

$$
z(t)=c_{1}+\dot{u} t+c_{2} e^{-(b / M) t} .
$$

The velocity of the free mass is then given by the derivative of (6) as

$$
\dot{z}(t)=\dot{u}-c_{2} \frac{b}{M} e^{-(b / M) t} .
$$

Equation (6) is a closed form solution of linearized equation (3). The solution of nonlinear equation (3) will be developed later since it requires a numerical approach.

The structure is modeled as a single-degree-of-freedom system consisting of a mass, damper, and spring without external forces (other than impacts with the free mass) as in Figure 1. Since the source of structural vibrations is the transfer of momenta during collisions, outside the time of the collisions, the structure vibrates as a free simple harmonic oscillator. The equation of structural motion is expressed as

$$
m \ddot{x}+c \dot{x}+k x=0 .
$$

For an underdamped case, (8) has the following solution:

$$
x(t)=c_{3} e^{-\zeta \omega_{n} t} \cos \omega_{d} t+c_{4} e^{-\zeta \omega_{n} t} \sin \omega_{d} t
$$

with $\zeta, \omega_{d}$, and $\omega_{n}$ having the usual interpretation and definition as damping coefficient, damped frequency, and natural frequency. The velocity of the structure at time $t$ can be calculated as

$$
\begin{aligned}
\dot{x}(t)= & -\omega_{d} c_{3} e^{-\zeta \omega_{n} t} \sin \omega_{d} t-\zeta \omega_{n} c_{3} e^{-\zeta \omega_{n} t} \cos \omega_{d} t \\
& +\omega_{d} c_{4} e^{-\zeta \omega_{n} t} \cos \omega_{d} t-\zeta \omega_{n} c_{4} e^{-\zeta \omega_{n} t} \sin \omega_{d} t .
\end{aligned}
$$


From (6), (7), (9), and (10), the displacements and velocities of the mass and structure at any given time can be found. Substituting these expressions into the equations for the conservation of total system momentum and the equation for the coefficient of restitution, the displacements and velocities immediately after impact can be evaluated. Each of the two masses, however, has two unknowns after impact. Thus the unknowns after impact are the displacement and velocity of both masses. Since only two equations can be developed from the conservation of momentum and the coefficient of restitution equation, the number of unknowns exceeds the number of equations by two. Salapaka et al. [25] also investigated a similar modeling approach. They developed the equation of a mass-spring system driven by a vibrating table which impacts the mass at a certain position repetitively. Faced with the same problem of excessive unknowns, they assumed that the displacements of each mass before and after impact remain the same, thus reducing the number of unknowns after impact to two [25].

The instants just before and just after impact will be denoted by $t_{k}$ and $t_{f}$. Since the impact occurs when the displacements of the two masses are the same, the displacements at time $t_{k}$ are given as

$$
x\left(t_{k}\right)=z\left(t_{k}\right)=x_{k} .
$$

The same notation is used for velocities. From the conservation of momentum, the total momentum of the two masses, $m$ and $M$, is conserved so that

$$
m \dot{x}_{f}+M \dot{z}_{f}=m \dot{x}_{k}+M \dot{z}_{k} .
$$

The second relevant impact equation is obtained from the coefficient of restitution [26] as

$$
\dot{x}_{f}-\dot{z}_{f}=e\left(\dot{z}_{k}-\dot{x}_{k}\right) .
$$

Equations (12) and (13) can be rewritten in matrix form as

$$
\left[\begin{array}{cc}
m & M \\
1 & -1
\end{array}\right]\left[\begin{array}{c}
\dot{x}_{f} \\
\dot{z}_{f}
\end{array}\right]=\left[\begin{array}{c}
m \dot{x}_{k}+M \dot{z}_{k} \\
e \dot{z}_{k}-e \dot{x}_{k}
\end{array}\right]
$$

or

$$
\left[\begin{array}{c}
\dot{x}_{f} \\
\dot{z}_{f}
\end{array}\right]=\frac{-1}{m+M}\left[\begin{array}{cc}
-1 & -M \\
-1 & m
\end{array}\right]\left[\begin{array}{c}
m \dot{x}_{k}+M \dot{z}_{k} \\
e \dot{z}_{k}-e \dot{x}_{k}
\end{array}\right] .
$$

From (15), the velocities after impact, $\dot{x}_{f}$ and $\dot{z}_{f}$, are

$$
\begin{gathered}
\dot{x}_{f}=\frac{1}{m+M}\left(m \dot{x}_{k}+M \dot{z}_{k}+e M \dot{z}_{k}-e M \dot{x}_{k}\right), \\
\dot{z}_{f}=\frac{1}{m+M}\left(m \dot{x}_{k}+M \dot{z}_{k}-e m \dot{z}_{k}+e m \dot{x}_{k}\right) .
\end{gathered}
$$

After impact, the displacements are assumed to be the same as before impact. From (11)

$$
\begin{aligned}
& x\left(t_{f}\right)=x\left(t_{k}\right)=x_{k}, \\
& z\left(t_{f}\right)=z\left(t_{k}\right)=x_{k} .
\end{aligned}
$$

Once the impact occurs, the momentum of the free mass is transmitted to the structure or vice versa. $c_{1}, c_{2}, c_{3}$, and $c_{4}$ of (6), (7), (9), and (10) need to be repeatedly calculated after each impact in order to obtain the new responses. Since the characteristics of the system responses are reset after each impact, the time right after the impact, $t_{f}$, is also reset to 0 to calculate $c_{1}, c_{2}, c_{3}$, and $c_{4}$. Substituting $t_{f}=0$ into (6), and (7),

$$
\begin{gathered}
z\left(t_{f}\right)=z(0)=c_{1}+c_{2}, \\
\dot{z}\left(t_{f}\right)=\dot{z}(0)=\dot{u}-\frac{b}{M} c_{2} .
\end{gathered}
$$

The displacements before and after impact are assumed to be the same, and the velocity of the free mass after the impact is calculated from (17). Equation (19) is equal to $x_{k}$ and $\dot{z}_{f}$, respectively

$$
\begin{gathered}
z(0)=c_{1}+c_{2}=x_{k}, \\
\dot{z}(0)=\dot{u}-\frac{b}{M} c_{2}=\dot{z}_{f} .
\end{gathered}
$$

From (20), $c_{1}$ and $c_{2}$ are calculated as

$$
\begin{gathered}
c_{1}=x_{k}-\frac{M}{b}\left(\dot{u}-\dot{z}_{f}\right), \\
c_{2}=\frac{M}{b}\left(\dot{u}-\dot{z}_{f}\right) .
\end{gathered}
$$

Applying the same assumption and (16) to (9) and (10),

$$
\begin{gathered}
x\left(t_{f}\right)=x(0)=c_{3}=x_{k}, \\
\dot{x}\left(t_{f}\right)=\dot{x}(0)=-\zeta \omega_{n} c_{3}+\omega_{d} c_{4}=\dot{x}_{f} .
\end{gathered}
$$

It then follows that $c_{3}$ and $c_{4}$ are obtained from (22) as

$$
\begin{gathered}
c_{3}=x_{k}, \\
c_{4}=\frac{1}{\omega_{d}}\left(\dot{x}_{f}+\zeta \omega_{n} x_{k}\right) .
\end{gathered}
$$

Substituting the calculated $c_{1}, c_{2}, c_{3}$, and $c_{4}$ into (6) and (9), the responses of the two masses after impact are expressed as

$$
\begin{aligned}
& z(t)=\left[x_{k}-\frac{M}{b}\left(\dot{u}-\dot{z}_{f}\right)\right]+\dot{u} t+\frac{M}{b}\left(\dot{u}-\dot{z}_{f}\right) e^{-(b / M) t}, \\
& x(t)=x_{k} e^{-\zeta \omega_{n} t} \cos \omega_{d} t+\frac{1}{\omega_{d}}\left(\dot{x}_{f}+\zeta \omega_{n} x_{k}\right) e^{-\zeta \omega_{n} t} \sin \omega_{d} t
\end{aligned}
$$

All the equations are developed based on the linearized drag force. Equation (24) holds until the next impact. After the next impact $c_{1}, c_{2}, c_{3}$, and $c_{4}$ should be recalculated according to new values of $x_{k}, \dot{x}_{k}, z_{k}$, and $\dot{z}_{k}$ obtained from the impact equations. 


\section{Numerical Simulations}

4.1. Linearized Drag Force. For the numerical simulations, there were two primary concerns: how to identify the instant of an impact, and how to define the impact condition. Since the basis of this repeated impact model is the exchange of momenta through impacts, the time and condition of the impact need to be defined as precisely as possible. The solution chosen to address this was to use a time stepping algorithm. In the beginning, $c_{1}, c_{2}, c_{3}$, and $c_{4}$ are calculated with the initial conditions. By substituting $c_{1}, c_{2}$, $c_{3}$, and $c_{4}$ into (24), the responses of the free mass and structure are obtained. Based on the calculated response, the relative displacement between the free mass and structure is compared to the impact condition at every time step. The impact condition is theoretically given by

$$
|x(t)-z(t)|=0 .
$$

The actual numerical simulation, however, cannot achieve enough precision to capture the exact impact condition. Therefore, a tolerance for the impact condition is required to allow for numerical round-off errors of the numerical simulation.

The tolerance should be considered carefully because too small tolerance has no meaning and a large tolerance makes impacts occur more often than they should. In the algorithm, the tolerance for the impact condition is set flexibly by the following equation:

$$
\Delta=\frac{1}{10} \min (|\delta x|,|\delta z|),
$$

where $\Delta$ is the impact condition such that $|x(t)-z(t)| \leq \Delta$ and $\delta x=\dot{x}(t) \Delta t$, and $\delta z=\dot{z}(t) \Delta t$. Furthermore, $\Delta t$ is the time step. The flexible impact condition has merit over a fixed one. When the responses of the two masses are very small, the fixed impact condition cannot capture some impacts. The flexible impact condition, however, is determined according to the size of the responses; therefore the impact condition is able to capture impacts whether the responses are slow or fast.

After the comparison of the relative displacement and the impact condition, if the relative displacement is larger than the impact condition, the same values of $c_{1}, c_{2}, c_{3}$, and $c_{4}$ will be forwarded to the next time step. Otherwise, the relative displacement is equal to or less than the impact condition and thus an impact takes place. Once the algorithm catches the impact, $c_{1}, c_{2}, c_{3}$, and $c_{4}$ are calculated based on the values from the previous time step and new conditions corresponding to the impact as explained in the previous section. In addition, the algorithm is performed on two different time scales: one is global time and the other is local time. The global time is the overall time starting with the beginning of the algorithm and the local time counts time duration between impacts; thus after each impact the local time is reset to 0 while the global time increases continuously. With 0 local time, new responses of the system based on the new $c_{1}, c_{2}, c_{3}$, and $c_{4}$ begin and evolve until next impact. This process keeps repeating until the system reaches the steady state.
The steady-state condition is also implemented in the algorithm to avoid infinitesimal impacts which occur at the end of the responses. The steady-state condition involves displacements, velocities, and accelerations. The algorithm verifies at every step whether or not both responses of the ice and structure satisfy the steady-state conditions. The steadystate conditions are given as
(i) $\max (|z(t)|,|x(t)|)<0.0001 \mathrm{~m}$,
(ii) $\max (|\dot{z}(t)|,|\dot{x}(t)|)<0.0001 \mathrm{~m} / \mathrm{s}$,
(iii) $\max (|\ddot{z}(t)|,|\ddot{x}(t)|)<0.0001 \mathrm{~m} / \mathrm{s}^{2}$.

The value of 0.0001 was chosen through trial and error for the steady-state condition. When the system reaches the steady state, the structure is constantly forced by the free mass, and so the equation of motion in the steady state is expressed as

$$
m \ddot{x}+c \dot{x}+k x=-b(\dot{z}-\dot{u})
$$

Since the system is in the steady state,

$$
k x=b \dot{u} .
$$

In the steady state condition, the two masses collide and move together; therefore the responses are given by

$$
x(t)=z(t)=\frac{b}{k} \dot{u}
$$

which is a constant (steady state) displacement since $\dot{u}$ is the (assumed) constant velocity of the flow. The algorithm replaces (24) with (28) when the steady-state condition is satisfied.

Figure 2 is plotted using the same structural properties in Table 1 which are obtained from [27]. The two free mass properties, mass, $M$, and area, $A$, are arbitrarily chosen as $1600 \mathrm{~kg}$ which is the same as the structural mass and $1 \mathrm{~m}^{2}$, respectively. The reason for this is as follows. If the free mass is much larger than the structural mass, then its displacement and velocity would be hardly affected by the collision with the structure and in fact repeated impacts would not occur. On the other hand, if the free mass is much smaller than that of the structure then the structure itself would hardly be affected by the impacts. Thus the most meaningful dynamics will occur when the two masses are relatively the same order of magnitude. For simplicity, we begin our analysis by choosing them to be the same, and later in the paper we investigate the effect of different choices of for the free mass.

The linearization factor, $|\dot{z}-\dot{u}|$, is set as $0.1 \mathrm{~m} / \mathrm{s}$ because it is the maximum difference between the free mass velocity and flow velocity which is set as $0.1 \mathrm{~m} / \mathrm{s}$. Therefore, the value of $b$ is

$$
b=\frac{1}{2} \rho C_{D} A|\dot{z}-\dot{u}|=49.95 \mathrm{~kg} / \mathrm{s},
$$

where $\rho$ is the density of water and $C_{D}$, the drag coefficient, is 1. The coefficient of restitution, another important parameter, is assumed to be 1 which implies no energy dissipation through impact. In addition, the initial displacement and 


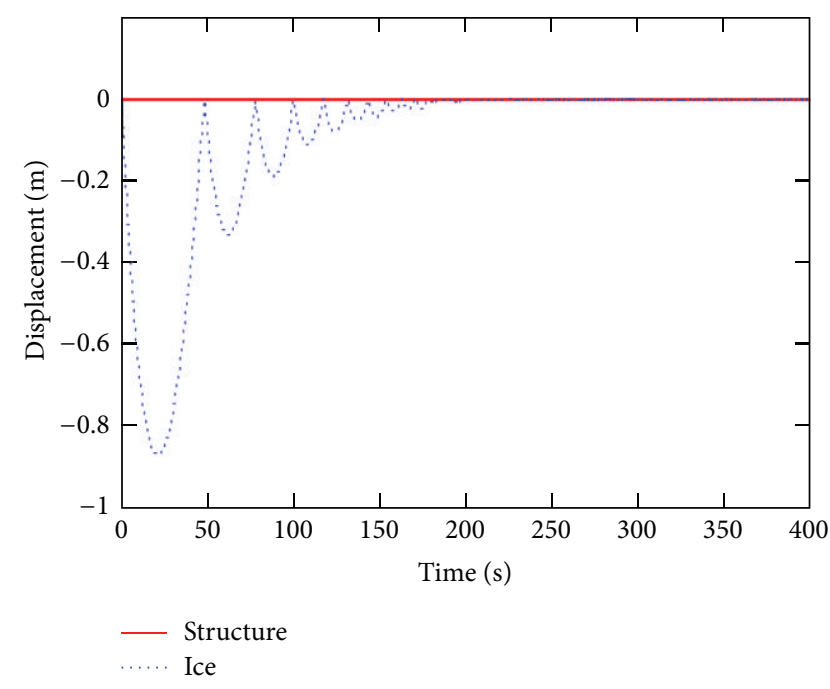

(a) Overall Responses

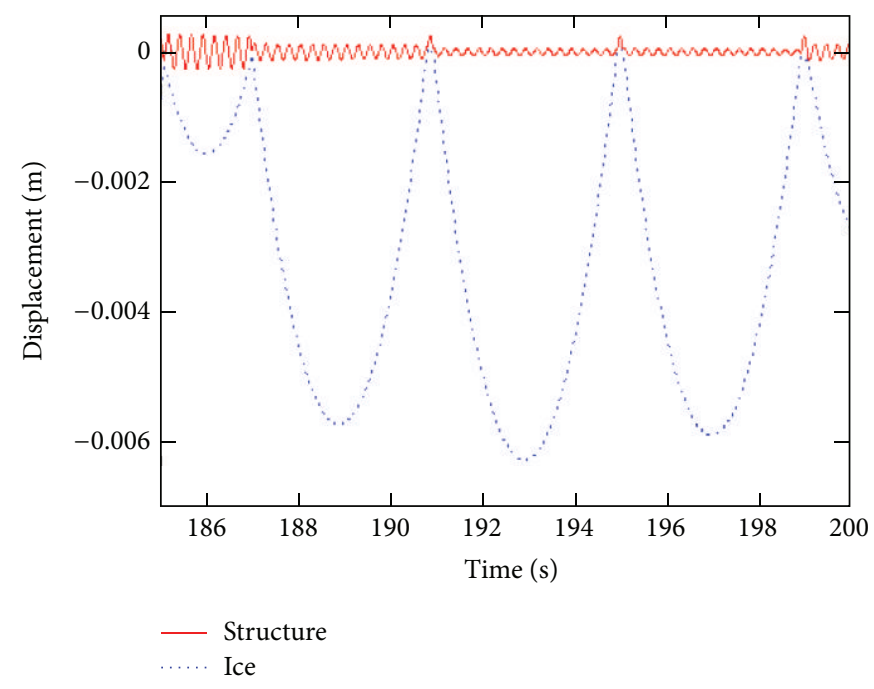

(b) Responses from 185 to 200 seconds

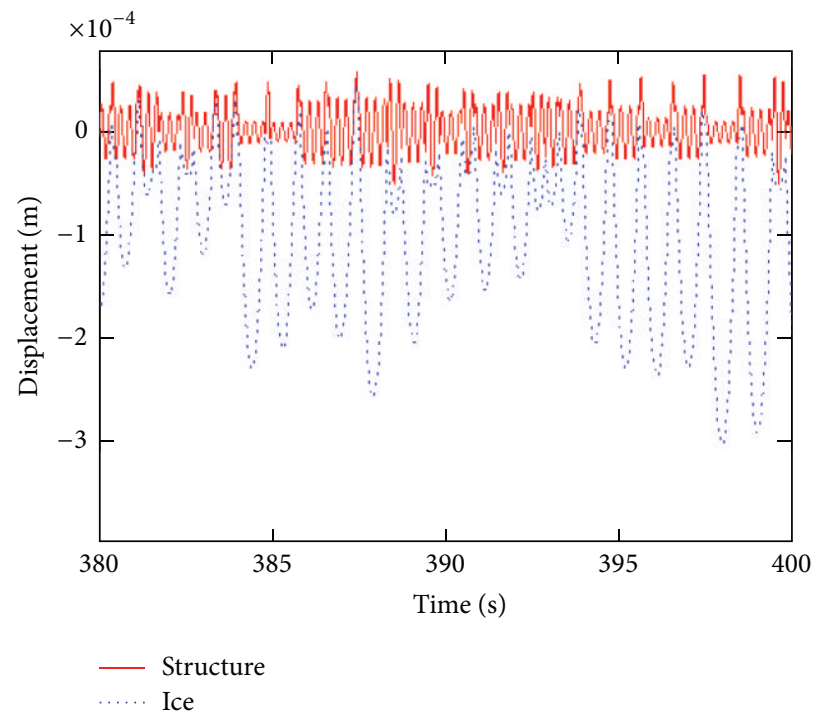

(c) Responses from 380 to 400 seconds

FIgURE 2: Responses of the ice and structure with a linearized drag force.

TABle 1: Parameters for simulations.

\begin{tabular}{lc}
\hline Parameter & Model structure \\
\hline$h(\mathrm{~mm})$ & 41 \\
$D(\mathrm{~mm})$ & 76 \\
$m(\mathrm{~kg})$ & 1600 \\
$c(\mathrm{kNs} / \mathrm{m})$ & 0.2 \\
$k(\mathrm{MN} / \mathrm{m})$ & 1 \\
$\ell(\mathrm{m})$ & 44.93 \\
$\sigma_{c}(\mathrm{MPa})$ & 1.4 \\
$x_{\max }(\mathrm{mm})$ & 20 \\
\hline
\end{tabular}

velocity of the free mass are $-0.01 \mathrm{~m}$ and $0.01 \mathrm{~m} / \mathrm{s}$, respectively, and $0 \mathrm{~m}$ and $0 \mathrm{~m} / \mathrm{s}$ for the structure. Figure 2 shows the response based on these parameters.
Since the motivation for this model was to attempt to explain IIV without analyzing specific ice-failure mechanisms, we attempt to compare our results to some measured IIV data. There is very little IIV data in the open literature, especially for displacement measurements. Only laboratory measurements are available from $[28,29]$ for displacement measurements. Since the structural data for their tests are not presented, it is inadequate to compare their experimental data to Figure 2 directly, but general vibration characteristics can be compared. Structural vibrations of IIVs become quasi-static vibrations at low ice velocities and steady-state vibrations at medium ice velocities [30] although there is no quantitative definition of low and high ice velocities. A quasi-static vibration is defined by Kärnä [30] as a transient response which is followed by its maximum response at the peak ice force and is not amplified by the dynamics of the structure. Figure 3(b) represents typical quasi-static 


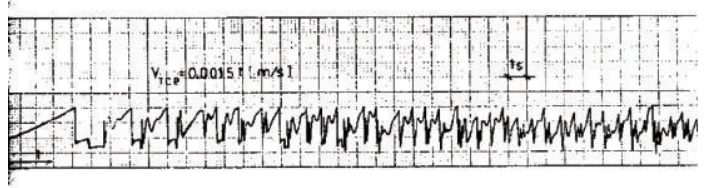

(a) $[28]$

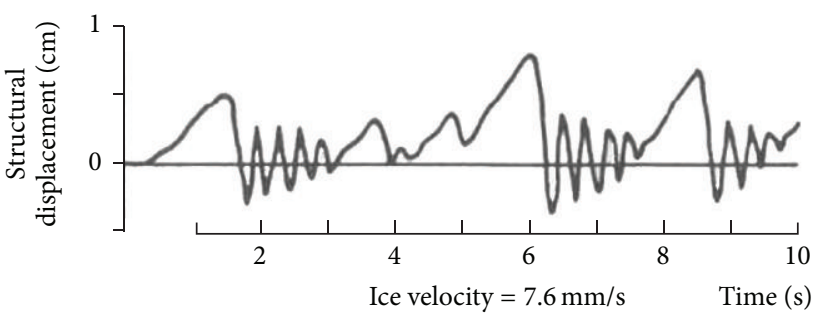

(b) [29]

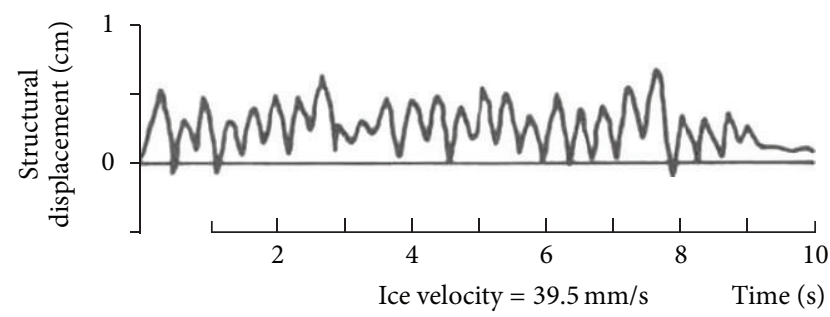

(c) $[29]$

FIgURE 3: Measured structural responses from the literature.

vibrations at ice velocity $7.6 \mathrm{~mm} / \mathrm{s}$ and Figure 3(c) shows the characteristic of a steady-state vibration at free mass velocity $39.5 \mathrm{~mm} / \mathrm{s}$. Figure 3(a), however, presents the displacement response although it is plotted at a lower free mass velocity than Figure 3(b). This demonstrates that the vibration characteristics of IIVs are not determined by the velocity of the ice alone but by involving all parameters.

As shown in Figure 3(a), actual IIVs display multiple vibration characteristics from quasi-static to steady-state vibrations even at the same ice velocity, contrary to the characteristic failure frequency model which predicts one type of characteristic vibration at one ice velocity. In this respect, the proposed impact model possesses an advantage over the characteristic failure frequency model. In an impact model, the velocity of the ice varies from time to time, which is more realistic because part of the ice (free mass) contacting the structure is deformed as the vibrations proceed. Figure 2 clearly shows multiple vibration characteristics. Figure 2(b) displays quasi-static vibrations similar to Figure 3(b). From 380 to 400 seconds, the vibrations become stable and steady state shown in Figure 2(c) which is close to the vibration characteristic of Figure 3(c). The vibration characteristics of the impact model, however, cannot be clearly distinguished for the entire duration; rather they show a mixture of the characteristics. Therefore, it is reasonable to conclude that the vibration characteristics of the impact IIV model are governed by the momenta of the ice and structure at the time of impact.

The weakness of modelling IIVs as repeated impacts as proposed in this paper model is how to model the relevant properties of the ice. The definitions of the ice mass and area in the impact IIV model imply that the mass and area directly affect the impact or interaction with the structure. Among the two ice properties, the influence of the ice area $A$ is directly related to the velocity of the flow because of the relation with $b$ given by $b=(1 / 2) \rho C_{D} A|\dot{z}-\dot{u}|$. It is inappropriate to simulate the linearized model with large flow velocities since then the linearized model would not be applicable.
The mass of the free mass plays an important role in this model. In order to understand its role, it will be investigated by conducting several numerical simulations. Figures 4, 5, and 6 are plotted with the same parameters of Figure 2 except with different masses which are proportional to the structural mass, 50 and 150 percent of $1600 \mathrm{~kg}$, so that the structural mass can be used as a benchmark. Including Figure 2, which is plotted with the free mass of 100 percent of the structural mass, the responses with three different free masses are compared. At the beginning of the responses, the heavier mass transfers higher momentum; thus impacts happen less frequently but with larger magnitudes. On the other hand, the lighter mass allows the structure to approach the steady-state vibration in relatively short time. In Figure 6 the magnitudes of the structural responses are not too different but the heavier mass induces more quasi-static vibrations for longer time. Therefore, it can be concluded that the free mass has a greater effect on determining the vibration characteristics than on the magnitude of the structural response.

Another important factor in this model is the coefficient of restitution. The coefficient of restitution also requires experimental research to be defined quantitatively. The conducted numerical simulations can only show how the coefficient of restitution influences the responses of the system. Figure 2 is plotted with the coefficient of restitution $e=1$ which is an unrealistic case. Figures 7,8 , and 9 are simulated based on the same parameters of Figure 2 but with different values of $e$. It becomes obvious that the factor that most influences the magnitudes of the vibrations is the coefficient of restitution rather than the velocities of the free mass. The system with $e=0.1$ approaches the steady state after the first impact. The system with $e=0.5$ not only approaches the steady-state vibration faster but also shows lower structural magnitudes than the system with $e=1$, Figure 2. Figure 8 indicates that the system with $e=0.5$ loses the majority of its momentum after approximately 54 seconds. This suggests that vibrations due to repeated impacts can be controlled by manipulating the coefficient 


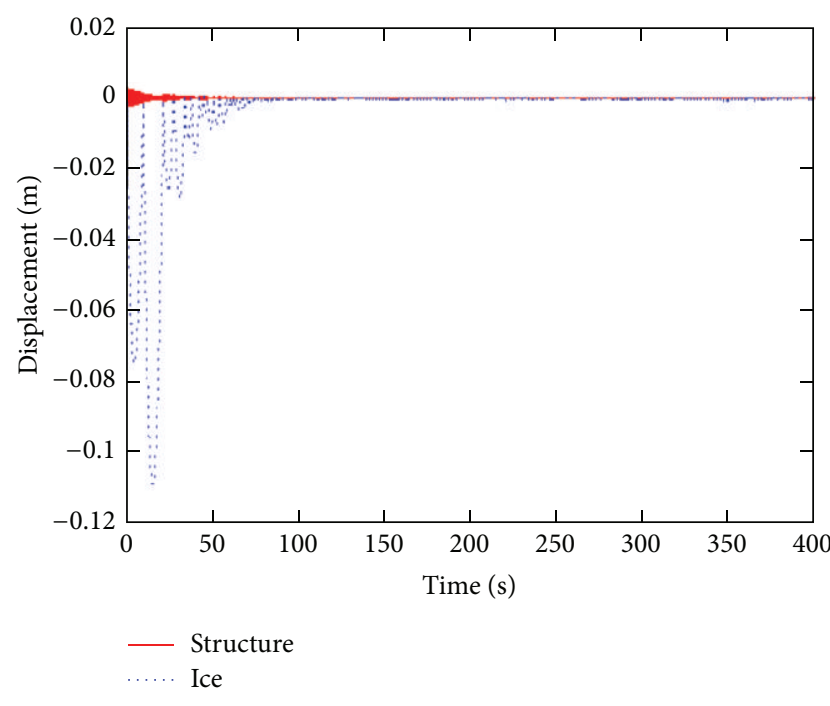

(a) Overall responses with $M=800$

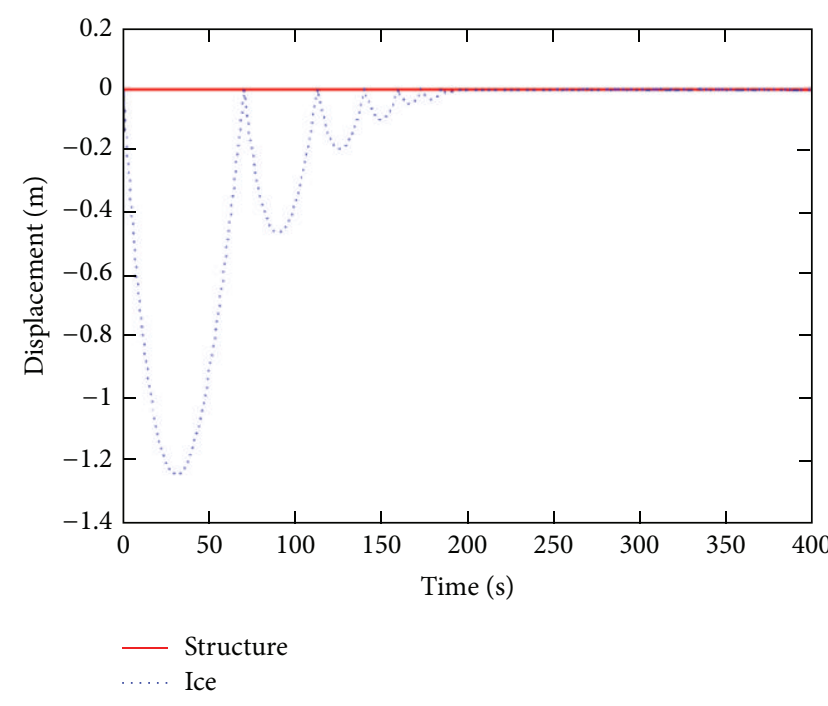

(b) Overall responses with $M=2400$

FIGURE 4: Responses with different ice masses 1.

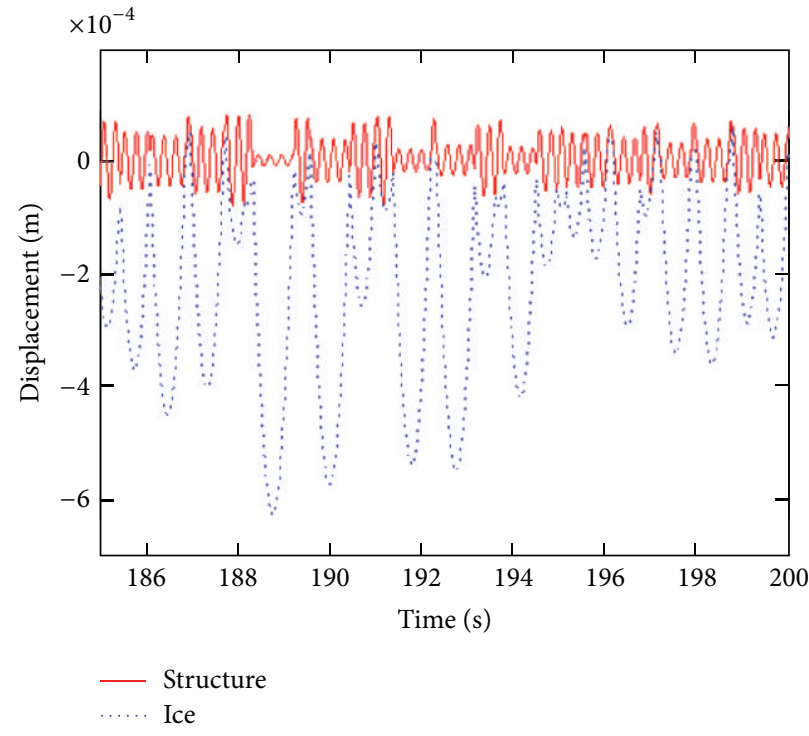

(a) Responses with $M=800$ from 185 to 200 seconds

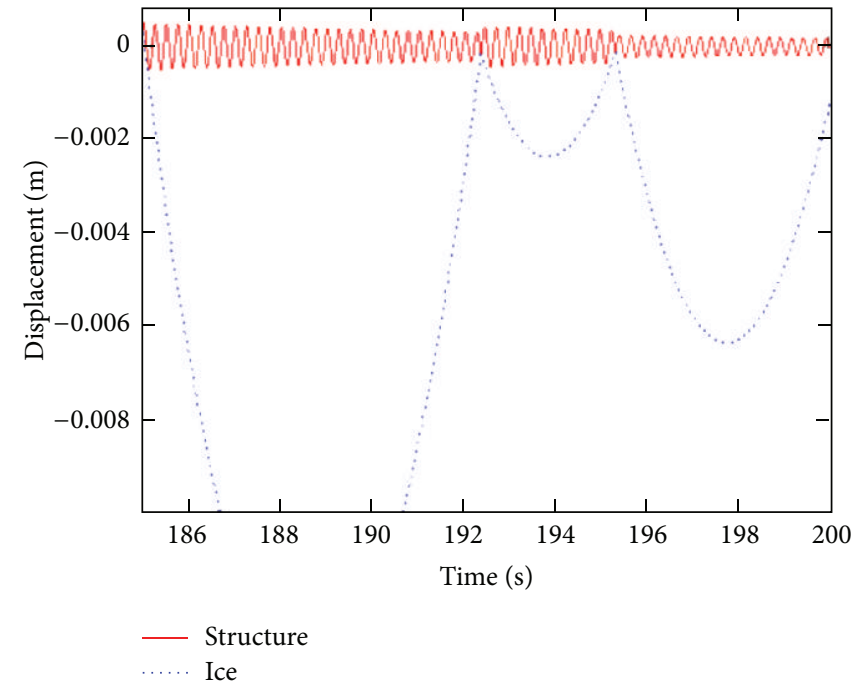

(b) Responses with $M=2400$ from 185 to 200 seconds

Figure 5: Responses with different ice masses 2.

of restitution via the properties of the contacting area. For instance, the magnitudes of vibrations can be significantly reduced by increasing the surface roughness of the structure.

4.2. Quadratic Drag Force. In the previous section, the model was developed based on the linearized drag force which assumes small relative velocity between the velocities of the free mass and structure. The linearized model, however, is definitely vulnerable to two factors, namely, when the interval between impacts exceeds several seconds, and when the velocity of the flow is no longer small. Figure 10 clearly shows the error between the models based on the linearized drag and quadratic drag as time passes. The displacement of linearized equations (3), and (6) is compared with numerically solved equation (3) by using the built-in ordinary differential equation solver of MathCad. The difference grows as time goes by, but at high flow velocity the difference decreases in Figure 10(b). This is because of the linearization factor, $|\dot{z}-\dot{u}|$, which calibrates the difference between the linear and quadratic models. The solution, however, to the quadratic drag force needs to be developed for a more precise simulation.

A numerical solution is needed to solve (3) but instead of using the built-in ordinary differential equation solver 


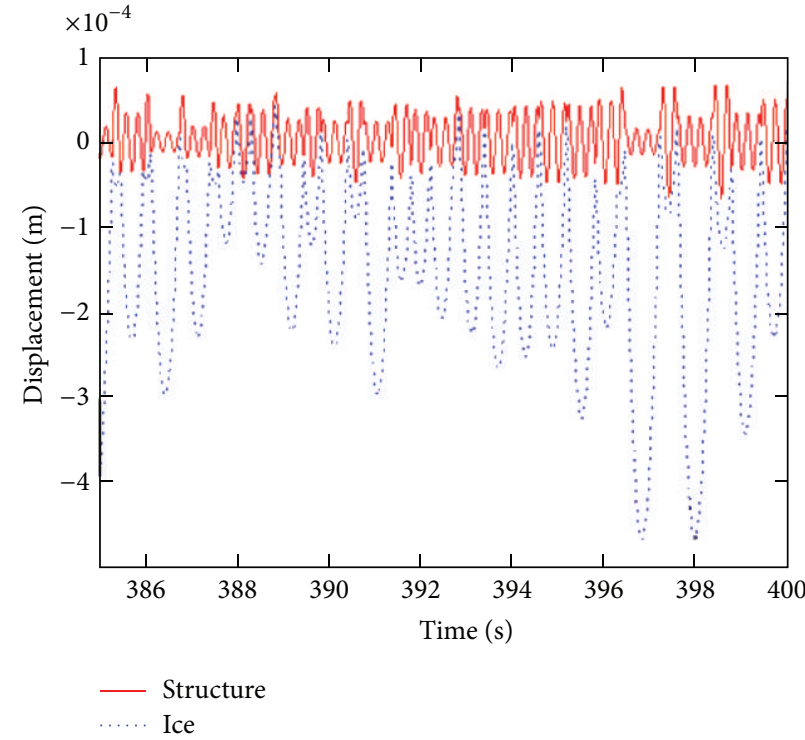

(a) Responses with $M=800$ from 385 to 400 seconds

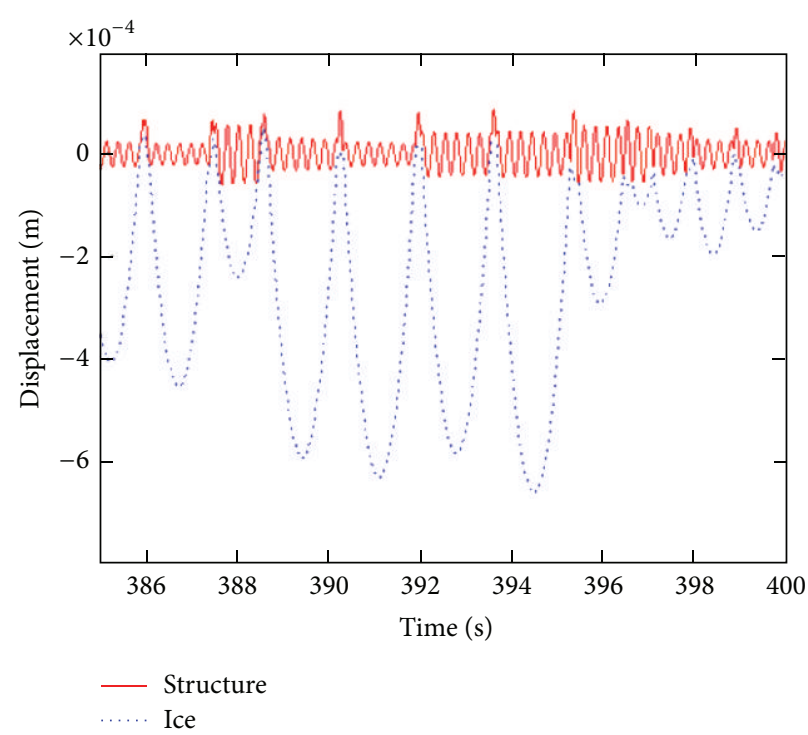

(b) Responses with $M=2400$ from 385 to 400 seconds

FIgURE 6: Responses with different ice masses 3.

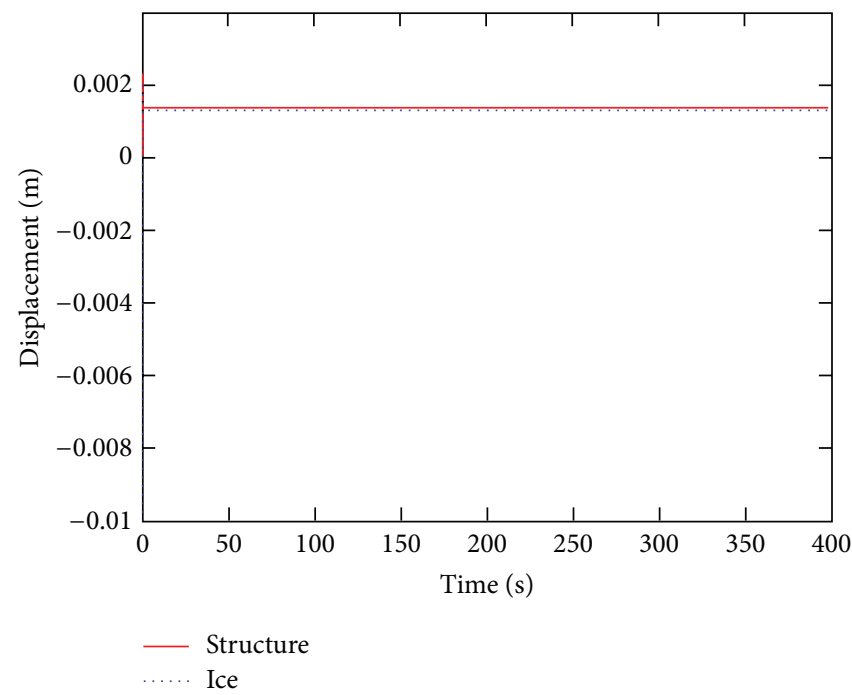

(a) Overall responses with $e=0.1$

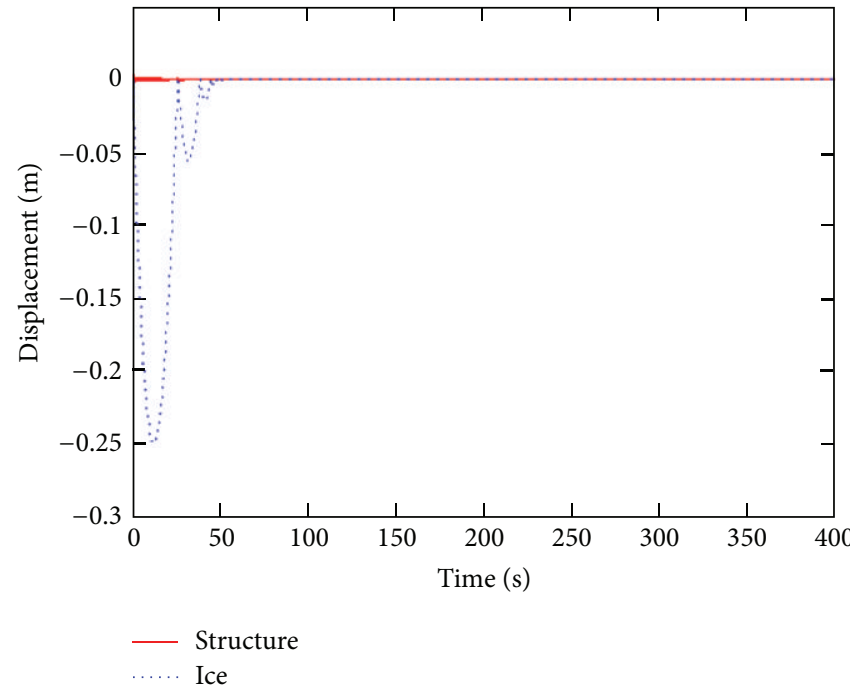

(b) Overall responses with $e=0.5$

FIgURE 7: Responses with different coefficients of restitution 1.

of MathCad, a simple numerical solution is developed for a faster algorithm. From (3),

$$
M \ddot{z}=-\frac{1}{2} \rho C_{D} A(\dot{z}-\dot{u})|\dot{z}-\dot{u}|=-B(\dot{z}-\dot{u})|\dot{z}-\dot{u}|
$$

or

$$
\ddot{z}=\frac{d \dot{z}}{d t}=-\frac{B}{M}(\dot{z}-\dot{u})|\dot{z}-\dot{u}|,
$$

where

$$
B=\frac{1}{2} \rho C_{D} A
$$

Using $i$ as a counter for the a number of time steps, the displacement of (34) can be expressed as

$$
z_{i}=z_{i-1}+\frac{d z_{i-1}}{d t} \Delta t=z_{i-1}+\dot{z}_{i-1} \Delta t
$$

in which $\Delta t$ is previously defined as a time step. $\dot{z}$ is given by

$$
\dot{z}_{i}=\dot{z}_{i-1}+\frac{d \dot{z}_{i-1}}{d t} \Delta t=\dot{z}_{i-1}+\ddot{z}_{i-1} \Delta t .
$$

By substituting (32) into (35),

$$
\dot{z}_{i}=\dot{z}_{i-1}+\left[-\frac{B}{M}\left(\dot{z}_{i-1}-\dot{u}\right)\left|\dot{z}_{i-1}-\dot{u}\right|\right] \Delta t .
$$




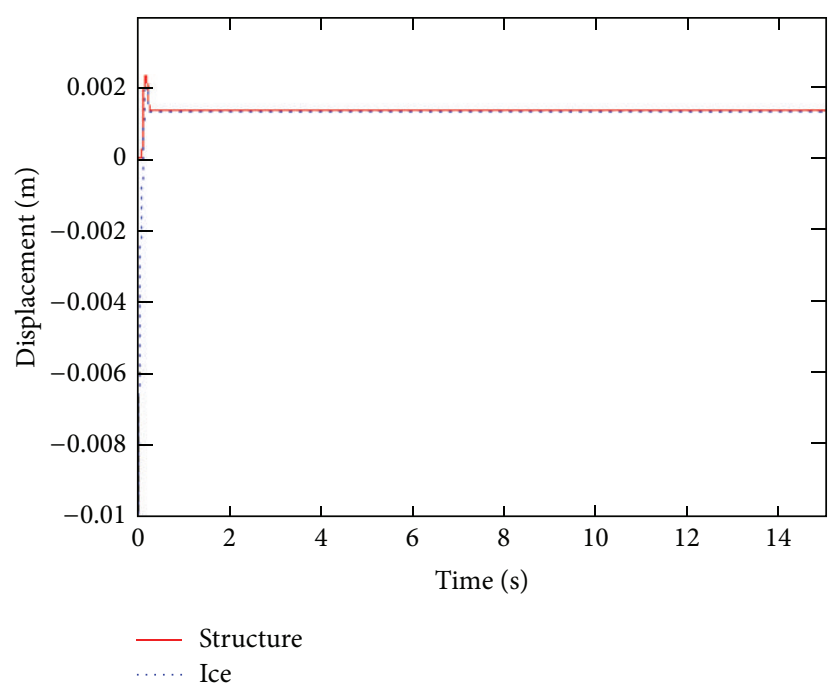

(a) Responses with $e=0.1$ from 0 to 15 seconds

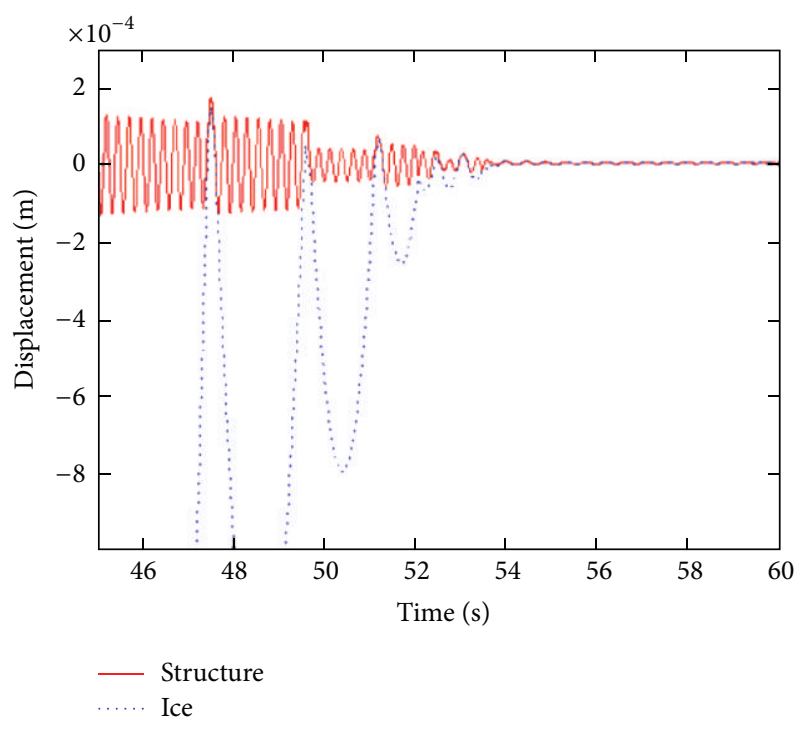

(b) Responses with $e=0.5$ from 45 to 60 seconds

FIGURE 8: Responses with different coefficients of restitution 2.

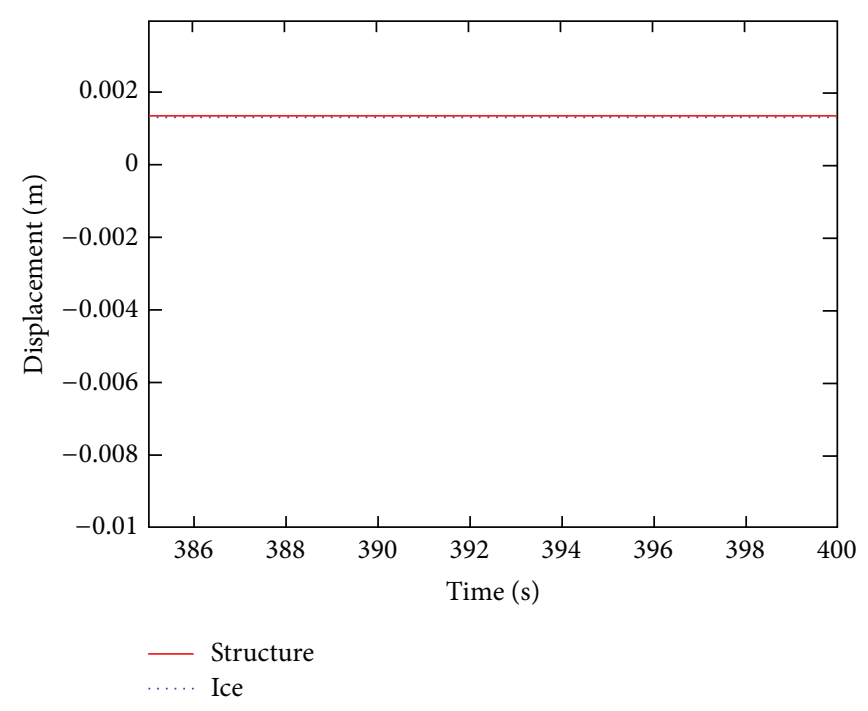

(a) Responses with $e=0.1$ from 385 to 400 seconds

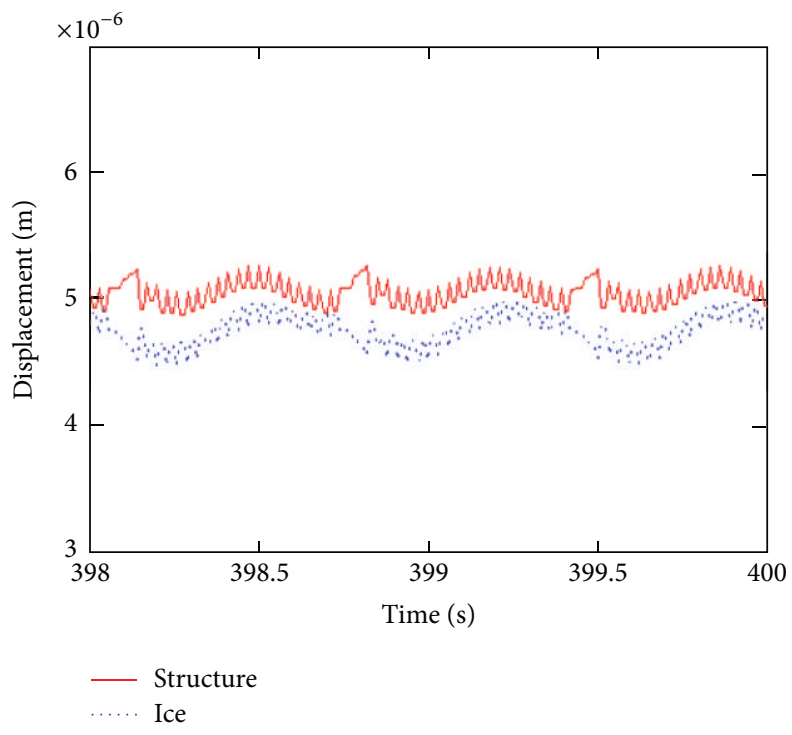

(b) Responses with $e=0.5$ from 398 to 400 seconds

FIGURE 9: Responses with different coefficients of restitution 3.

Given the initial displacement and velocity of the free mass, the displacement can be numerically solved by using (34) and (36) as simultaneous equations. The simulation results of (34) and (36) are also plotted in Figure 10. At the high flow velocity, Figure 10(b), (34), and (36) predict the displacement close enough to the one evaluated with the built-in ordinary differential equation solver. In the algorithm of the numerical simulation, (34) and (36) replace (6) and (7).

Using the same parameters as those used in Figure 2 but with a different flow velocity, the responses of the model with the quadratic drag force are compared with the one with the linearized drag force in Figure 11. At high flow velocities, over $1 \mathrm{~m} / \mathrm{s}$, the quadratic drag force model approaches steady-state vibration more quickly than the linearized drag force model because at high flow velocity the quadratic drag induces more damping while it transfers more forces than the linearized drag. In Figure 12 the quadratic drag force model exhibits the characteristic of the steadystate vibration while the linearized drag force model still displays quasi-static vibration. As velocities increase, which corresponds to flow velocities, increasing vibrations approach the steady state. The linearized drag force model, however, does not show a steady-state vibration until 400 seconds in Figure 12. The linearization factor allows the linearized 


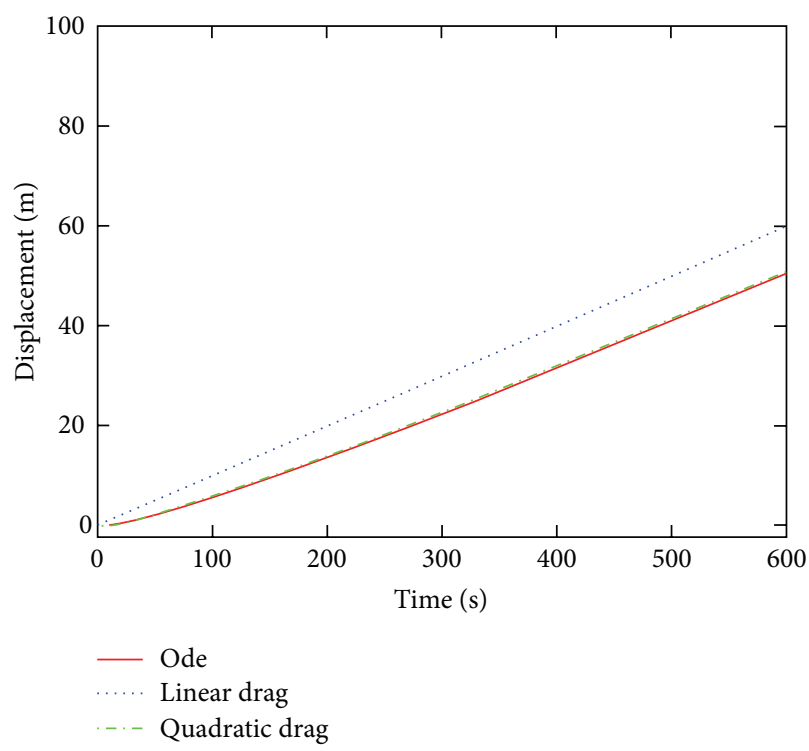

(a) Flow velocity $0.1 \mathrm{~m} / \mathrm{s}$

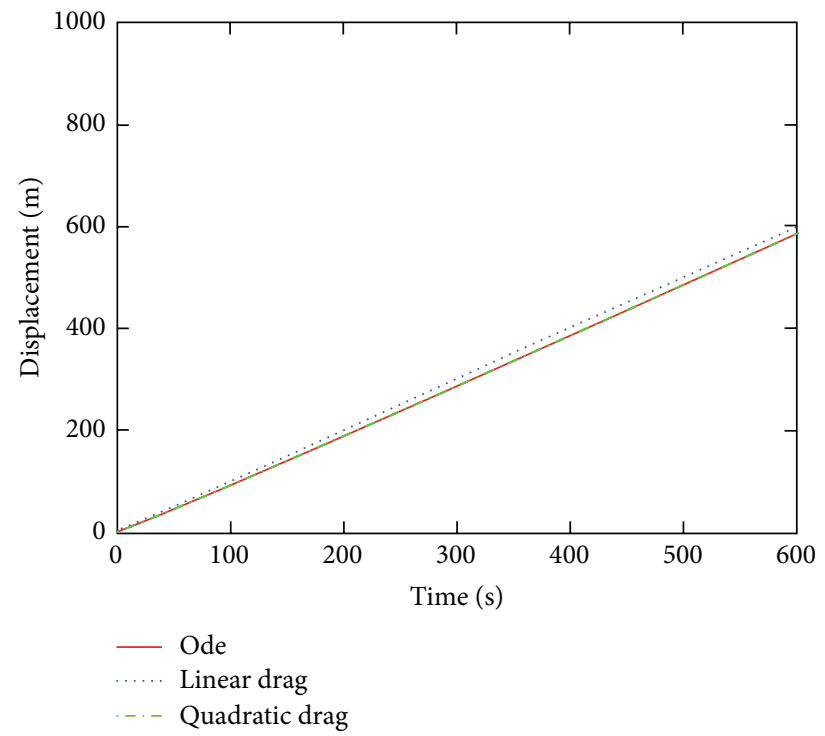

(b) Flow velocity $1 \mathrm{~m} / \mathrm{s}$

FIGURE 10: Comparison of the linearized and quadratic Drag.

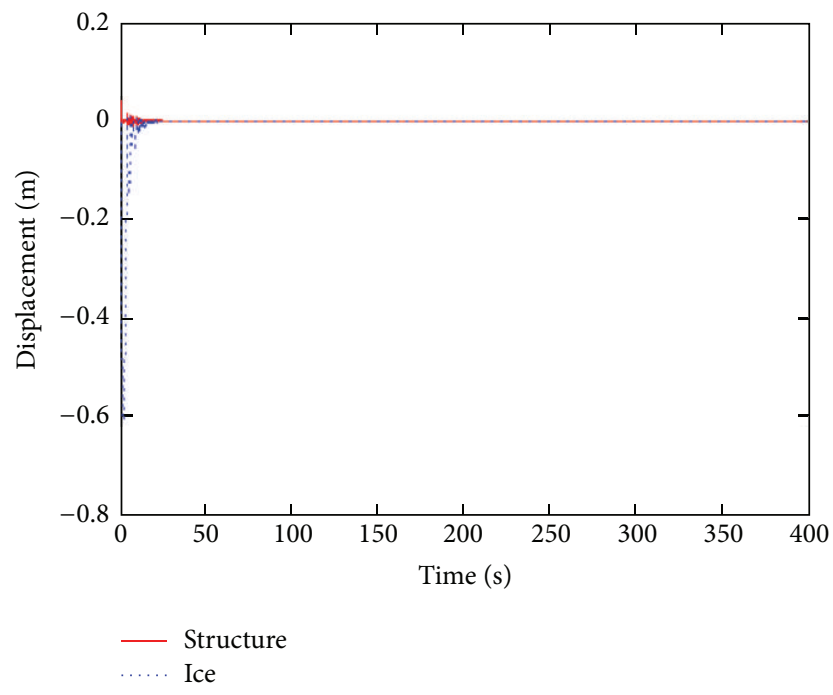

(a) Overall responses with the quadratic drag

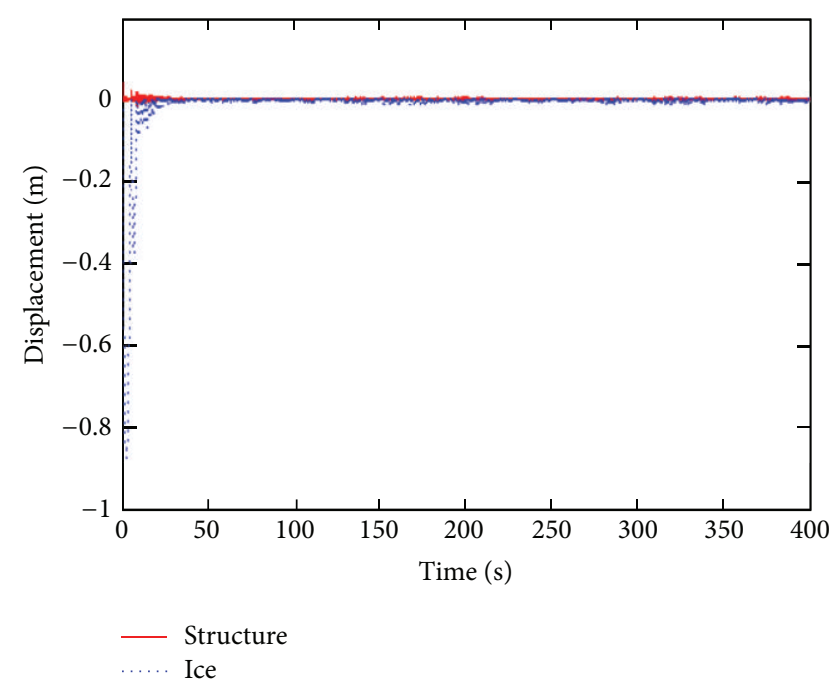

(b) Overall responses with the linear drag

FIGURE 11: Responses with the quadratic and linear drags 1.

drag force model to follow the quadratic drag force model in the beginning, but when the system is in a series of low velocity impacts, the linearization factor causes a difference. The linearized drag force model, therefore, is inappropriate to simulate the repeated impact model at high flow velocities.

Since the free mass area, $A$, plays a similar role as the flow velocity in the model, the model with wide area is also simulated with the quadratic drag force. The influence of the drag force on the response of the free mass increases as the size of the area increases because the area is related to the drag force by (1), $f_{D}=-(1 / 2) \rho C_{D} A(\dot{z}-\dot{u})^{2}$. Figure 13 is plotted with the area $A=2 \mathrm{~m}^{2}$ the flow velocity $\dot{u}=0.1 \mathrm{~m} / \mathrm{s}$ and the rest of parameters are the same as the parameters used in Figure 11. The flow velocity of Figure 13 is identical to that of Figure 2 but the responses quickly approach the steadystate vibrations as if they are driven by high flow velocity. Figure 13(b) also shows a similar vibration characteristic to Figure 12(a) because both flow velocity and area influence the drag force in the impact model. Therefore, the area can be another controlling factor of the vibrations. Since the area is related to the size of the contacting area of the structure, reducing the contacting area has a similar effect to that of reducing the flow velocity.

In the previous section, the roles of the free mass and the coefficient of restitution have been defined by changing 


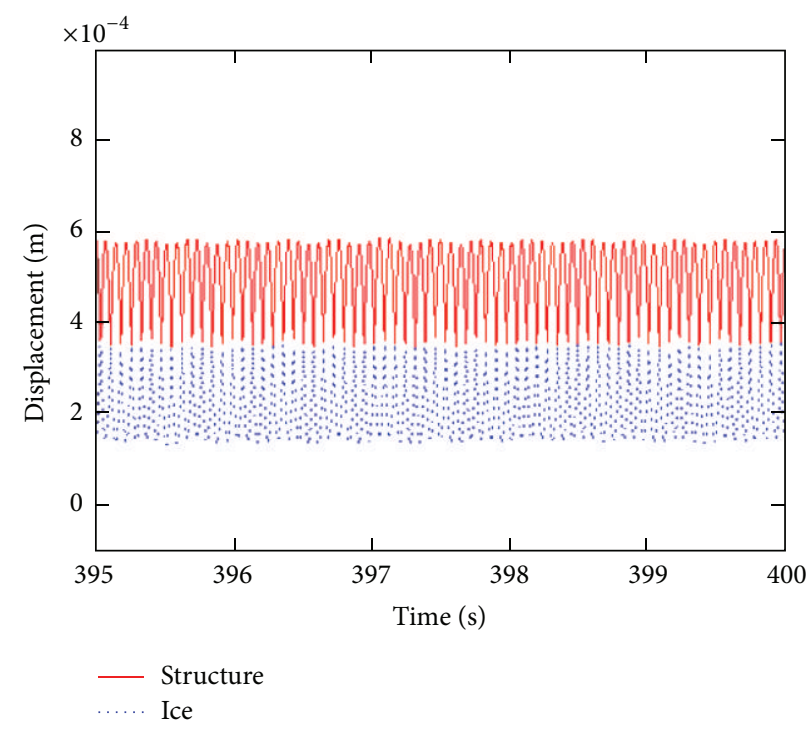

(a) Responses with the quadratic drag from 395 to 400 seconds

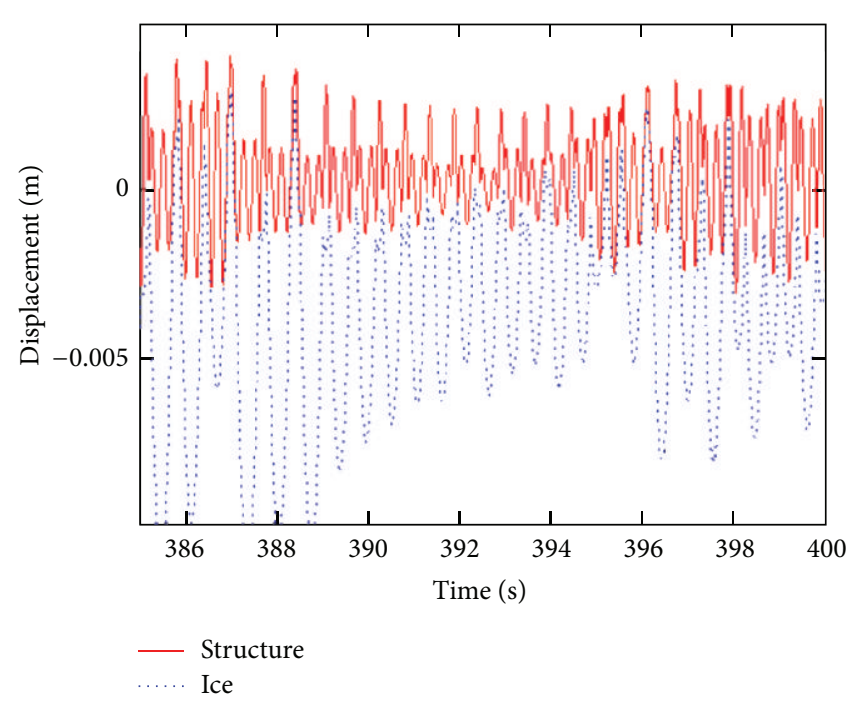

(b) Responses with the linear drag from 385 to 400 seconds

FIGURE 12: Responses with the quadratic and linear drags 2.

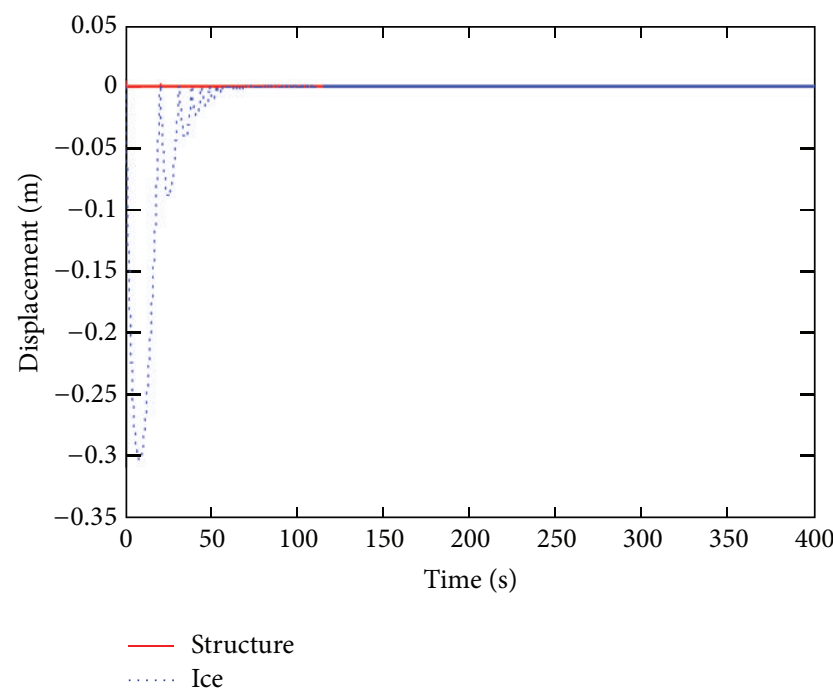

(a) Overall responses with $A=2$

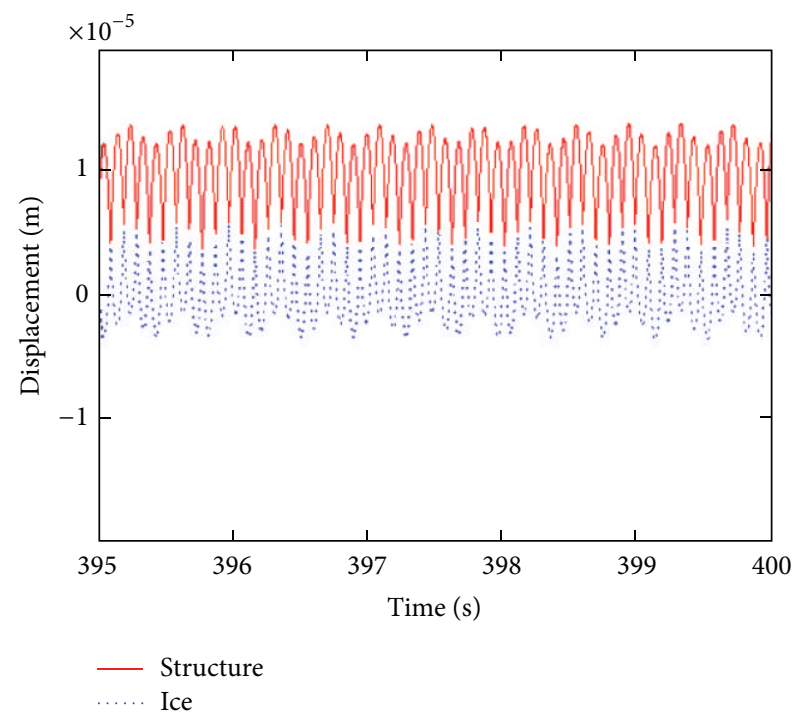

(b) Responses with $A=2$ from 395 to 400 seconds

Figure 13: Responses with $A=2 \mathrm{~m}^{2}$.

relevant parameters. To verify if those definitions still hold with the quadratic drag force, the impact model with the quadratic drag is plotted with change in free mass and the coefficient of restitution. Figures 14, 15, and 16 are plotted on the same parameters used in Figures 2, 4(a), and 4(b), respectively, except that they are based on the quadratic drag force. The responses of Figures 14, 15, and 16 prove that the defined role of the free mass holds with the quadratic drag force. The responses of the system with heavier free mass, Figure 16(b), is still unstable while the system with the free mass $M=800 \mathrm{~kg}$, Figure 15(b), is in the steady-state vibration. The quadratic drag force, however, induces more drag force; thus the system responses tend to be stabilized faster than those with the linearized drag forces.

Figures 17 and 18 are plotted on the same conditions with Figures $7(\mathrm{a})$ and $7(\mathrm{~b})$, respectively, but with the quadratic drag force. The coefficient of restitution also governs the magnitudes of responses in the impact model with the quadratic drag force; however, the system with the quadratic drag force is more sensitive to change in the coefficient of restitution than the one with the linearized drag force. The system with $e=0.5$, Figure 18 (b), loses the momentum at approximately 43 seconds under the influence of the quadratic drag force while the system with the linearized drag force and $e=0.5$, 


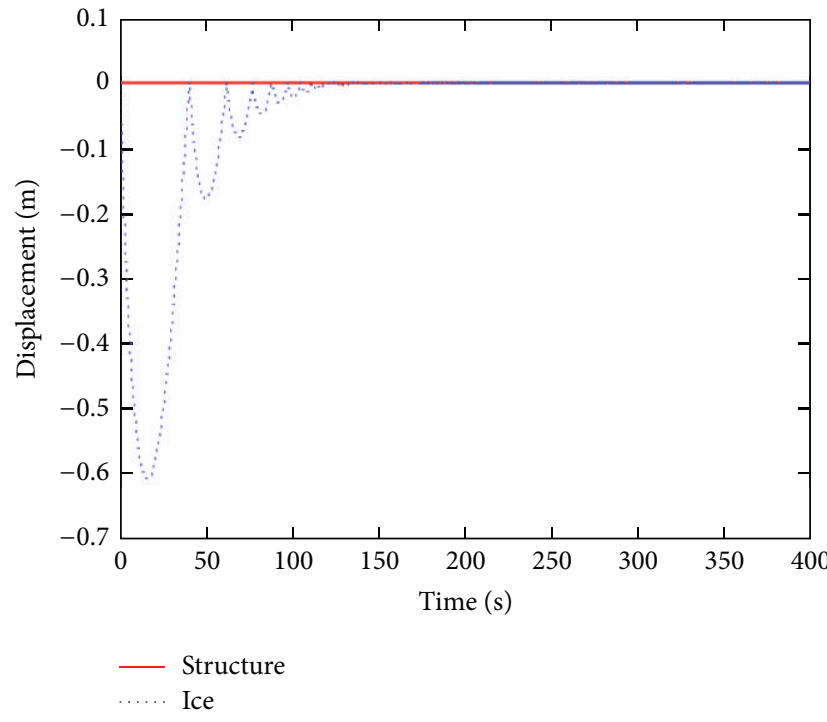

(a) Overall responses with $M=1600$

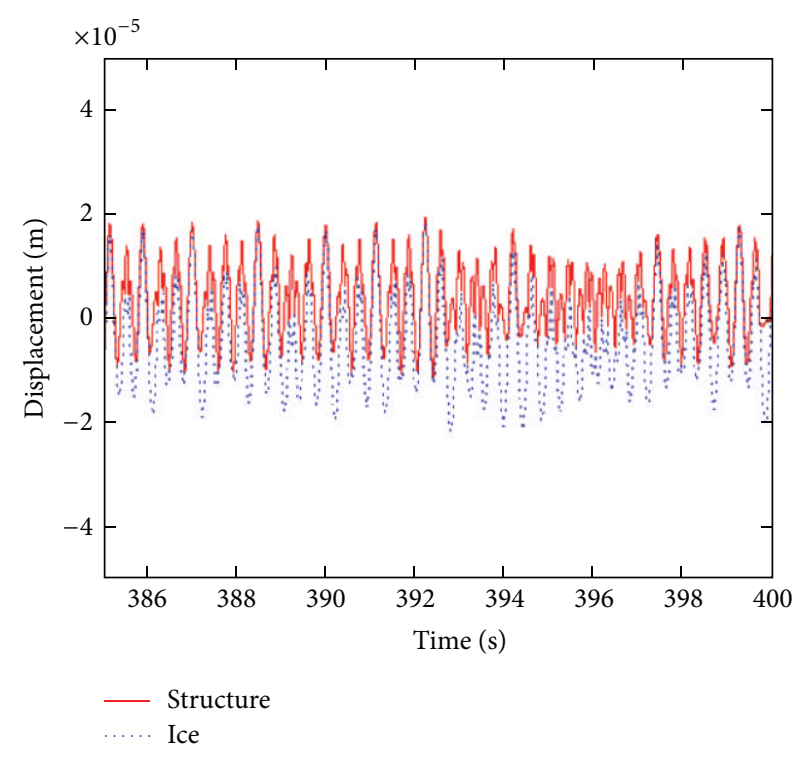

(b) Responses with $M=1600$ from 385 to 400 seconds

FIGURE 14: Responses with $M=1600$.

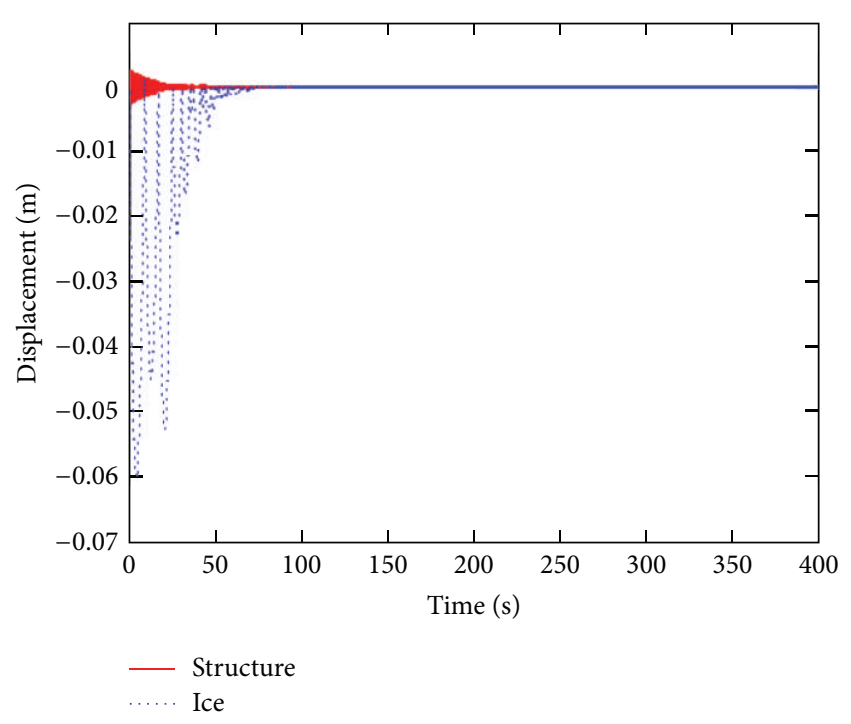

(a) Overall responses with $M=800$

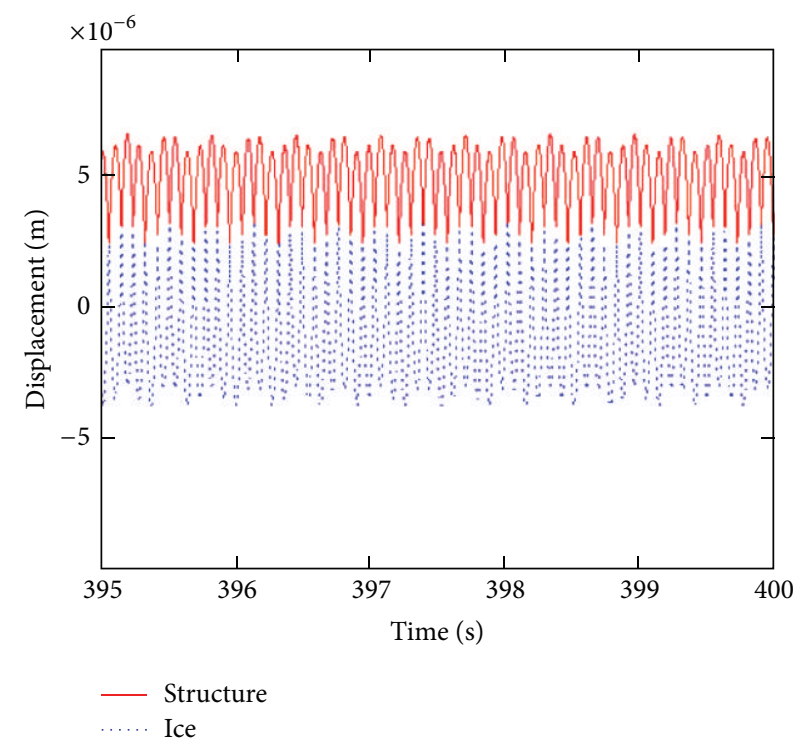

(b) Responses with $M=800$ from 395 to 400 seconds

FIgURE 15: Responses with $M=800$.

Figure $8(\mathrm{~b})$, loses the majority of its momentum at approximately 54 seconds. This is again because the quadratic drag force dissipates energy more quickly than the linearized drag force. However, it is demonstrated in Figures 17 and 18 that the coefficient of restitution plays the same role in both quadratic and linearized drag forces.

\section{Resonance Conditions}

One of the main objectives of the analysis aims to find a resonance condition (which is often referred to as lock-in when investigating IIVs). The structural vibration frequency increases as the free mass velocity increases but when lockin occurs, the structural frequency remains at the natural frequency even with increasing free mass velocity [6]. In the impact model, however, the velocities of the free mass cannot be the primary lock-in condition because the velocities of the free mass are not fixed.

One possible interpretation of modelling IIVs with the impact model is that it considers the forces to be a series of discrete impulses as shown in Figure 19(a) while other IIV models consider them as continuous periodic forces. 


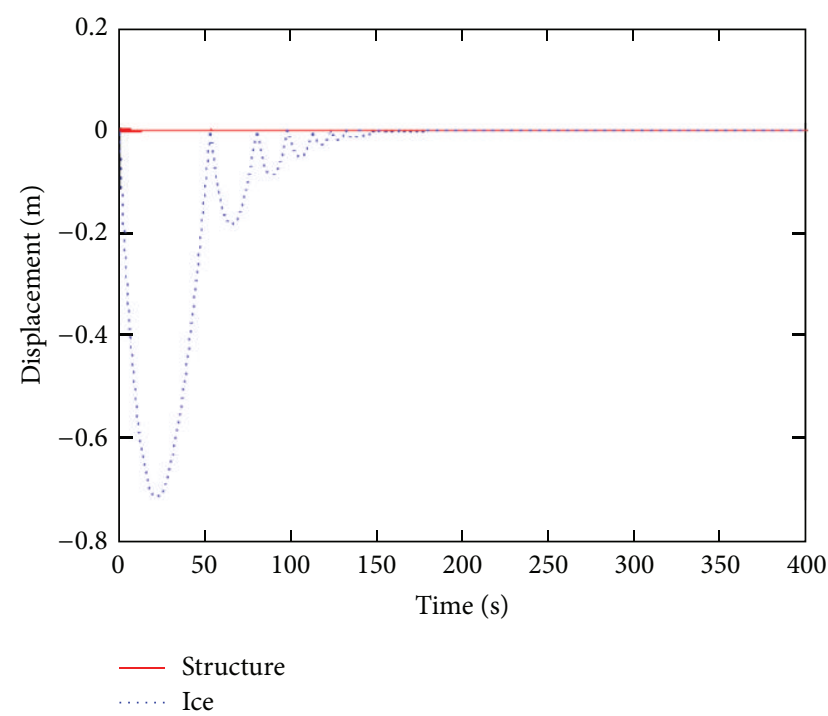

(a) Overall responses with $M=2400$

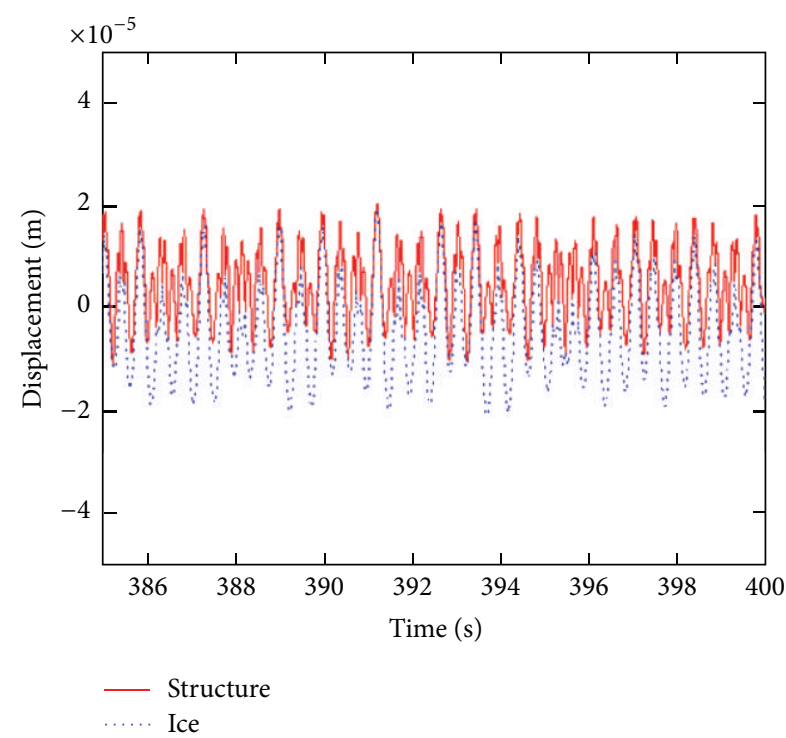

(b) Responses with $M=2400$ from 385 to 400 seconds

FIGURE 16: Responses with $M=2400$.

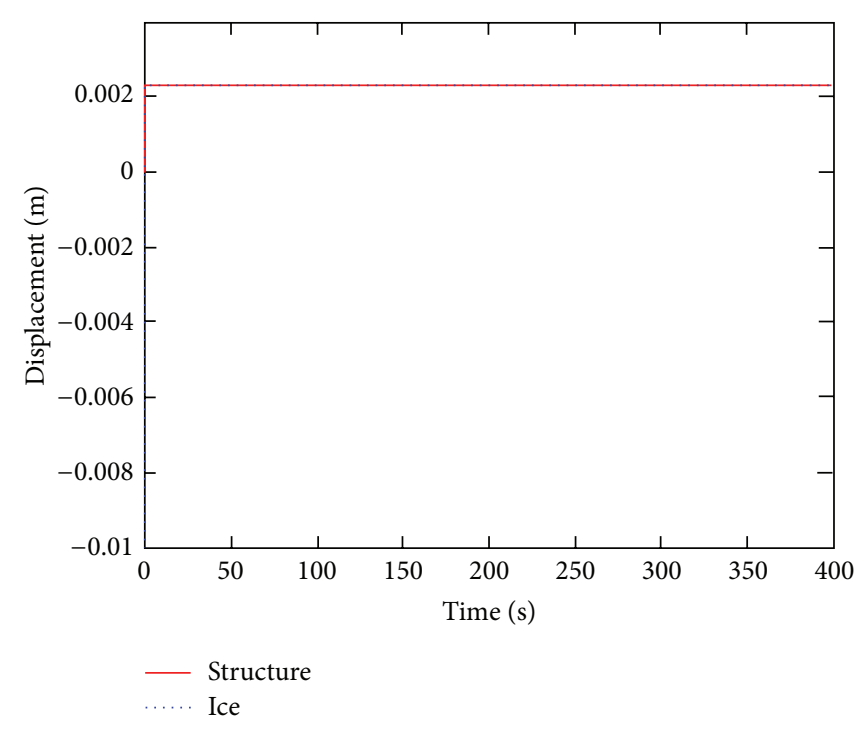

(a) Overall responses with $e=0.1$

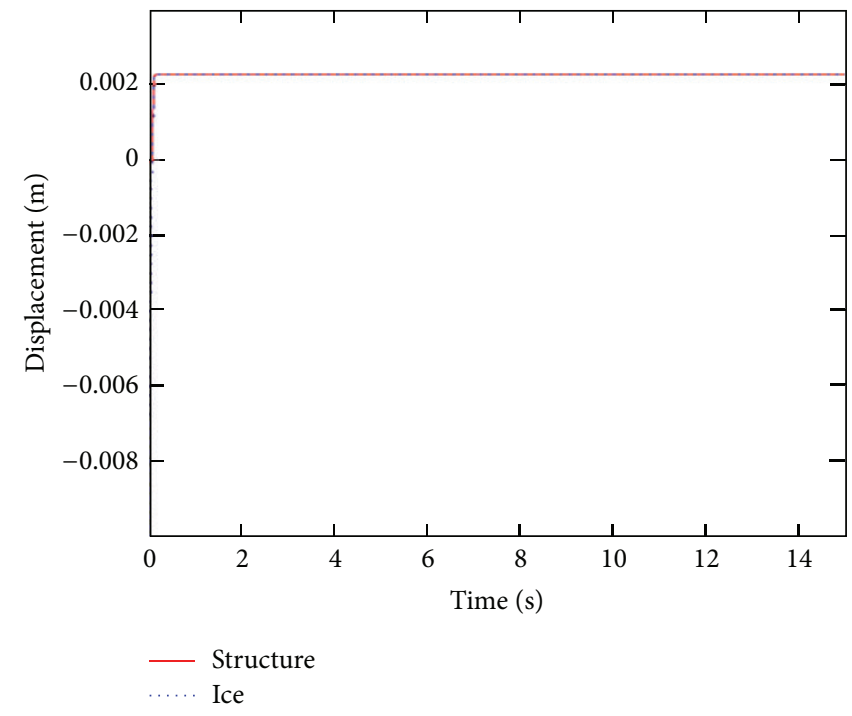

(b) Responses with $e=0.1$ from 0 to 15 seconds

FIGURE 17: Responses with $e=0.1$.

In fact, the impact IIV model proposes a way to calculate the strength of each impulse and the times between impulses and these depend on the momenta of the ice and structure as well as the coefficient of restitution for the collision. Under certain conditions, it is possible for the discrete impulses of Figure 19(a) to approach the regular periodic series of impulses of Figure 19(b) which is suspected to cause the lockin condition.

This view of the impact IIV model can be analyzed by using a Fourier transform [31]. There are several definitions of the Fourier transform (FT) that may be used and in this paper; the chosen version of the FT is given by

$$
\widehat{F}(\omega)=\int_{-\infty}^{\infty} f(t) e^{-i \omega t} d t
$$

The corresponding inverse Fourier transform is then given by

$$
f(t)=\frac{1}{2 \pi} \int_{-\infty}^{\infty} \widehat{F}(\omega) e^{i \omega t} d \omega .
$$




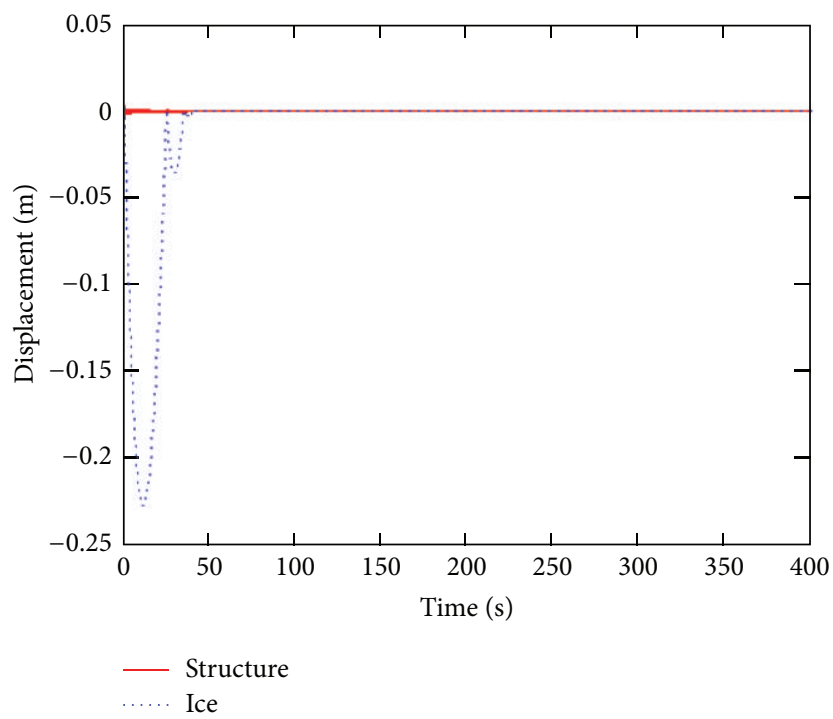

(a) Overall responses with $e=0.5$

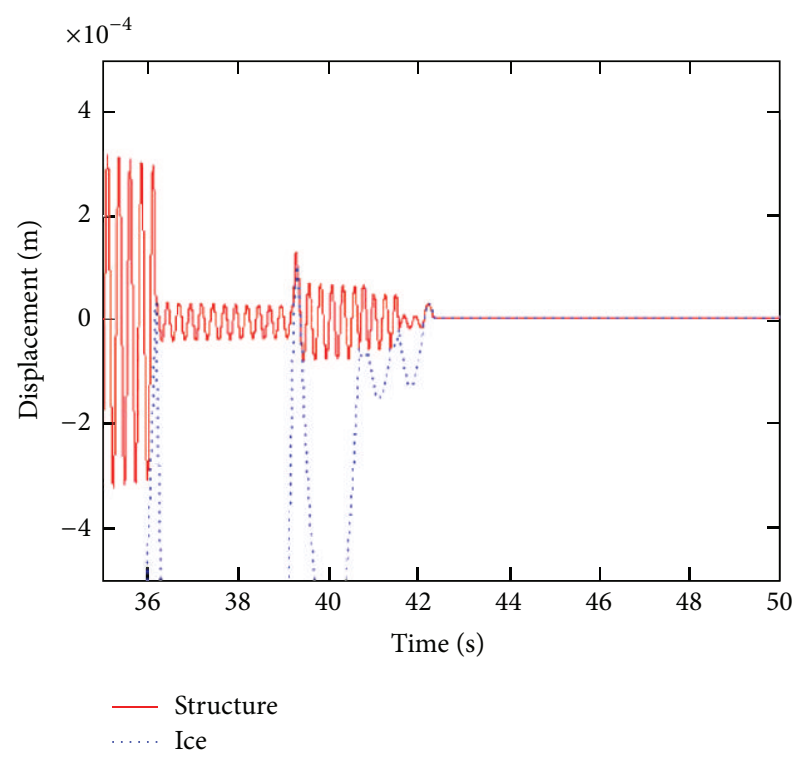

(b) Responses with $e=0.5$ from 35 to 50 seconds

FIGURE 18: Responses with $e=0.5$.

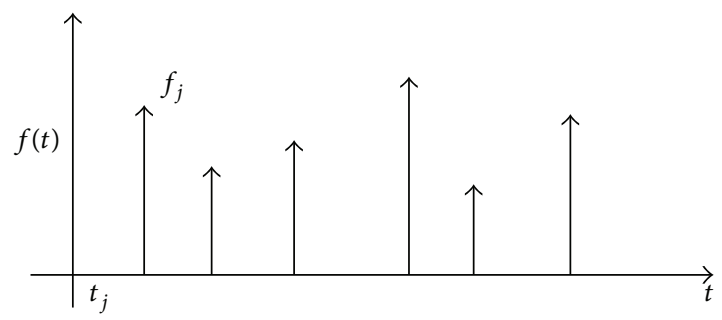

(a) Random impulses

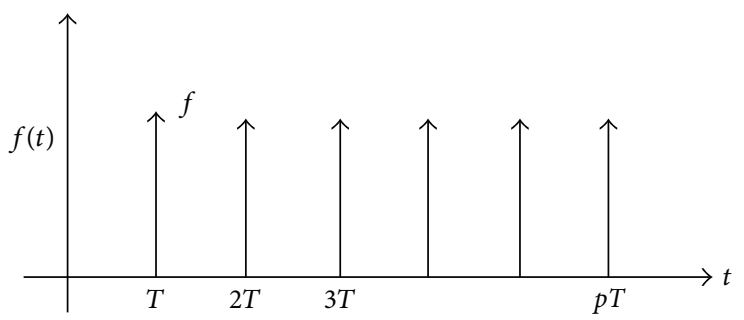

(b) Uniform impulses

FIGURE 19: Ice forces as a series of impulses.

The discrete ice forces of Figure 19(a) can be expressed as a series of impulses as

$$
f(t)=\sum_{j=1}^{\infty} f_{j} \delta_{D}\left(t-t_{j}\right),
$$

where $\delta_{D}$ indicates the Dirac delta function and $f_{j}$ indicates the strength of each impact. If the sizes and intervals of the impulses are uniform as shown in Figure 19(b), (39) is rewritten as

$$
f(t)=\sum_{p=1}^{\infty} f \delta_{D}(t-p T) .
$$

In reality, the repeated impacts will not all have the same strength or occur at a fixed interval; however it is sufficient for the situation of Figure 19(a) to approach that of Figure 19(b) for this analysis to be relevant.

Equation (40) is often called a comb function. The Fourier transform of the comb function is given [32] by

$$
\widehat{F}(\omega)=2 \pi \sum_{N=1}^{\infty} f \delta_{D}\left(\omega-\frac{2 \pi N}{T}\right) .
$$

For a typical single-degree of freedom forced vibrations problem,

$$
\ddot{x}+2 \zeta \omega_{n} \dot{x}+\omega_{n}^{2} x=\sum_{p=1}^{\infty} f \delta_{D}(t-p T) .
$$

Taking Fourier transforms of both sides and rearranging give

$$
\widehat{X}(\omega)=2 \pi \frac{\sum_{N=1}^{\infty} f \delta_{D}(\omega-2 \pi N / T)}{\left(-\omega^{2}+2 \zeta \omega_{n} \omega i+\omega_{n}^{2}\right)} .
$$

Taking the inverse Fourier transforms to find the structure response in time gives

$$
x(t)=f \sum_{N=1}^{\infty} \frac{e^{i(2 \pi N / T) t}}{\left(-4 \pi^{2} N^{2} / T^{2}+i \zeta \omega_{n}(4 \pi N / T)+\omega_{n}^{2}\right)} .
$$

Equation (44) experiences a resonance when the denominator of any of the terms in the sum goes to zero, that is when $\omega_{n}=2 \pi N / T$, where $N$ is an integer larger than 0 . This says that the structure will experience a resonance when the natural frequency is an integral multiple of the forcing frequency, 
as would be expected. In the previous numerical simulations, this condition has been observed in several cases, Figures 12(a) and 13(b). For instance, the structural frequency of Figure $13(\mathrm{~b})$ is $2 \pi / T=2 \pi / 0.5=12.57 \mathrm{rad} / \mathrm{s}$. The natural frequency of the structure is $\sqrt{k / m}=\sqrt{1000000 / 1600}=$ $25 \mathrm{rad} / \mathrm{s}$. With $N=2$ the structural frequency is close to the natural frequency. The result is what is seen in Figure 13(b). The two masses vibrate at the same frequency of $2 \pi / T$ which is half of the natural frequency. The structural frequency remains at that level, which is lock-in, and so does the frequency of the free mass response.

Thus, the impact model successfully predicts the existence of a lock-in condition and also predicts that it can occur when the frequency of the structure and free mass impacts are synchronized together at a multiple of the natural frequency of the structure. The question of the precise conditions necessary for the frequency of the impacts to occur at a multiple of the natural frequency still remains open.

\section{Summary and Conclusions}

This paper analyzes the vibrations caused by repeated impacts of a flow-driven free mass onto a second structure. Conservation of linear momentum and the coefficient of restitution are used to characterize the nature of the impacts between the two masses. This model is referred to as the impact model. The properties of the impact model were investigated through numerical simulations. The mass of the free mass is one of the factors determining the vibration characteristics since it is related to the momentum of each impact. This model is proposed to explain the nature of ice-induced vibrations without requiring a microscopic model of the mechanism of ice failure. The magnitudes of the vibrations are governed in part by the coefficient of restitution, which when used to explain IIVs, encapsulates all ice properties mentioned in other IIV models. In addition to the ice properties, the coefficient of restitution also governs the interaction between the structure and the free mass. The free mass is used to represent the ice when modelling IIVs. The last property of the impact model is that of the free mass area which influences the dissipation of the system momenta through the drag force. A free mass with a small area shows the same behavior of a mass driven by lower flow velocity.

Therefore, through the impact model, two factors which control the characteristics of IIVs have been proposed, the ice area and the coefficient of restitution. By changing the size of the contact area between the ice and structure, the driving force of the ice can be reduced as if the ice were driven by lower flow velocities. Furthermore, by changing factors related to the coefficient of restitution such as the surface roughness or shape of the structure, IIVs can be reduced to avoid the lock-in condition which occurs when the frequencies of the structure and ice impacts are synchronized at a multiple of the natural frequency of the structure. In addition to the inclusion of the effect of flow, the new approach to ice forces through the impact model permits the answering of questions which originate from the weak theoretical points of existing IIV models.
Particular strengths of the model are that it qualitatively matches observed results and in particular that a mechanism of ice failure is not required in order to perform simulations and predictions. It also proposes a simple mechanism to explain the potentially destructive resonance lock-in conditions.

\section{References}

[1] J. P. Dempsey, "Research trends in ice mechanics," International Journal of Solids and Structures, vol. 37, no. 1-2, pp. 131-153, 2000.

[2] K. A. Blenkarn, "Measurement and analysis of ice forces on cook inlet structures," in Proceedings of the Offshore Technology Conference (OTC'70), pp. 365-378, 1970.

[3] Y. Toyama, T. Sensu, M. Minami, and N. Yashima, "Model tests on ice-induced self-excited vibration of cylindrical structures," in Proceedings of the 7th International Conference on Port and Ocean Engineering under Arctic Conditions, vol. 2, pp. 834-844, 1983.

[4] M. Ranta and R. Räty, "On the analytic solution of ice-induced vibrations in a marine pile structure," in Proceedings of the 7th International Conference on Port and Ocean Engineering under Arctic Conditions, vol. 2, pp. 901-908, 1983.

[5] A. Barker, G. Timco, H. Gravesen, and P. Vølund, "Ice loading on Danish wind turbines-part 1: dynamic model tests," Cold Regions Science and Technology, vol. 41, no. 1, pp. 1-23, 2005.

[6] D. S. Sodhi, "Ice-induced vibrations of structures," Special Report 89-5, International Association for Hydraulic Research Working Group on Ice Forces, 1989.

[7] D. S. Sodhi and C. E. Morris, "Characteristic frequency of force variations in continuous crushing of sheet ice against rigid cylindrical structures," Cold Regions Science and Technology, vol. 12, no. 1, pp. 1-12, 1986.

[8] D. S. Sodhi, C. E. Morris, and G. F. N. Cox, "Dynamic analysis of failure modes on ice sheets encountering sloping structures," in Proceedings of the 6th International Conference on Offshore Mechanics and Arctic Engineering, vol. 4, pp. 281-284, 1987.

[9] H. R. Peyton, "Sea ice forces," in Proceedings of the Conference on Ice Pressure against Structures, 1966.

[10] C. R. Neill, "Dynamic ice forces on piers and piles. an assessment of design guidelines in the light of recent research," Canadian Journal of Civil Engineering, vol. 3, no. 2, pp. 305-341, 1976.

[11] G. Matlock, W. Dawkins, and J. Panak, "Analytical model for ice-structure interaction," Journal of the Engineering Mechanics Division, pp. 1083-11092, 1971.

[12] M. M. Määttänen, "Ice-induced vibrations of structures selfexcitation," Special Report 89-5, International Association for Hydraulic Research Working Group on Ice Forces, 1989.

[13] M. M. Määttänen, "Numerical simulation of ice-induced vibrations in offshore structures," in Proceedings of the 14th Nordic Seminar on Computational Mechanics, 2001, Keynote lecture.

[14] J. Xu and L. Wang, "Ice force oscillator model and its numerical solutions," in Proceedings of the 7th International Conference on Offshore Machanics and Arctic Engineering, pp. 171-176, 1988.

[15] G. Tsinker, Marine Structures Engineering, Chapman-Hall, New York, NY, USA, 1st edition, 1995.

[16] G. Huang and P. Liu, "A dynamic model for ice-induced vibration of structures," Journal of Offshore Mechanics and Arctic Engineering, vol. 131, no. 1, Article ID 011501, 6 pages, 2009. 
[17] R. Frederking and J. Schwarz, "Model tests of ice forces on fixed and oscillating cones," Cold Regions Science and Technology, vol. 6, no. 1, pp. 61-72, 1982.

[18] K. Hirayama and I. Obara, "Ice forces on inclined structures," in Proceedings of the 5th International Offshore Mechanics and Arctic Engineering, pp. 515-520, 1986.

[19] Q. Yue and X. Bi, "Full-scale test and analysis of dynamic interaction between ice sheet and conical structure," in Proceedings of the 14th International Association for Hydraulic Research (IAHR) Symposium on Ice, vol. 2, 1998.

[20] Q. Yue and X. Bi, "Ice-induced jacket structure vibrations in Bohai Sea," Journal of Cold Regions Engineering, vol. 14, no. 2, pp. 81-92, 2000.

[21] Y. Qu, Q. Yue, X. Bi, and T. Kärnä, "A random ice force model for narrow conical structures," Cold Regions Science and Technology, vol. 45, no. 3, pp. 148-157, 2006.

[22] X. Liu, G. Li, R. Oberlies, and Q. Yue, "Research on short-term dynamic ice cases for dynamic analysis of ice-resistant jacket platform in the Bohai Gulf," Marine Structures, vol. 22, no. 3, pp. 457-479, 2009.

[23] X. Liu, G. Li, Q. Yue, and R. Oberlies, "Acceleration-oriented design optimization of ice-resistant jacket platforms in the Bohai Gulf," Ocean Engineering, vol. 36, no. 17-18, pp. 1295-1302, 2009.

[24] B. Munson, D. Young, and T. Okiishi, Fundamentals of Fluid Mechanics, John Wiley \& Sons, Ames, Iowa, USA, 4th edition, 2002.

[25] S. Salapaka, M. V. Salapaka, M. Dahleh, and I. Mezic, "Complex dynamics in repeated impact oscillators," in Proceedings of the 37th IEEE Conference on Decision and Control (CDC '98), pp. 2053-2058, December 1998.

[26] A. Bedford and W. Fowler, Engineering Mechanics: Dynamics, Addison Wesley Longman, Inc., Menlo Park, Calif, USA, 2nd edition, 1999.

[27] T. Kärnä and R. Turunen, "Dynamic response of narrow structures to ice crushing," Cold Regions Science and Technology, vol. 17, no. 2, pp. 173-187, 1989.

[28] E. Eranti, F. D. Hayes, M. M. Määttänen, and T. T. Soong, "Dynamic ice-structure interaction analysis for narrow vertical structures," in Proceedings of the 6th International Conference on Port and Ocean Engineering under Arctic Conditions, vol. 1, pp. 472-479, 1981.

[29] L. Y. Shih, "Analysis of ice-induced vibrations on a flexible structure," Applied Mathematical Modelling, vol. 15, no. 11-12, pp. 632-638, 1991.

[30] T. K. Kärnä, "Steady-state vibrations of offshore structures," Hydrotechnical Construction, vol. 28, no. 9, pp. 446-453, 1994.

[31] C. W. de Silva, Vibration: Fundamentals and Practice, CRC Press LLC, Boca Raton, Fla, USA, 1st edition, 1999.

[32] H. J. Weaver, Applications of Discrete and Continuous Fourier Analysis, John Wiley \& Sons, New York, NY, USA, 1st edition, 1983. 


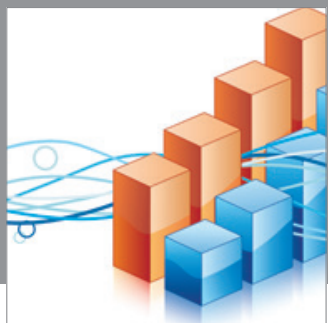

Advances in

Operations Research

mansans

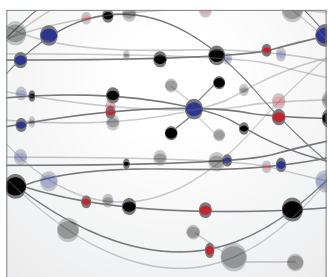

The Scientific World Journal
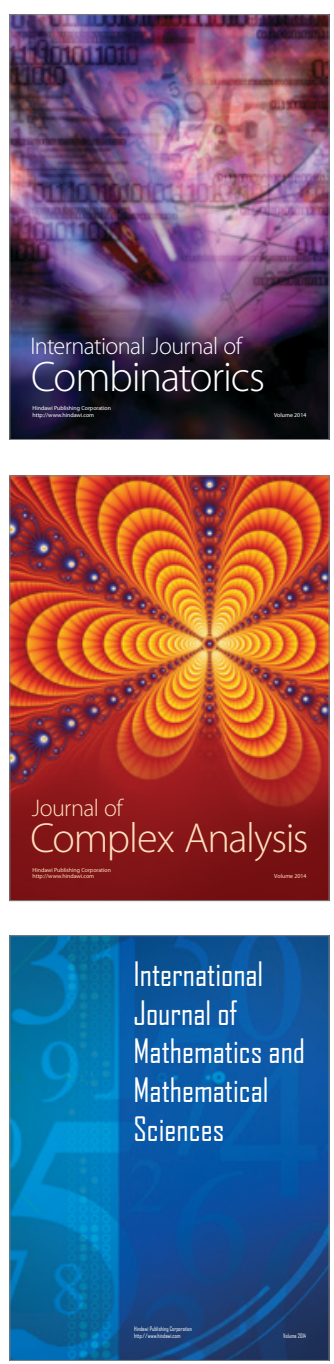
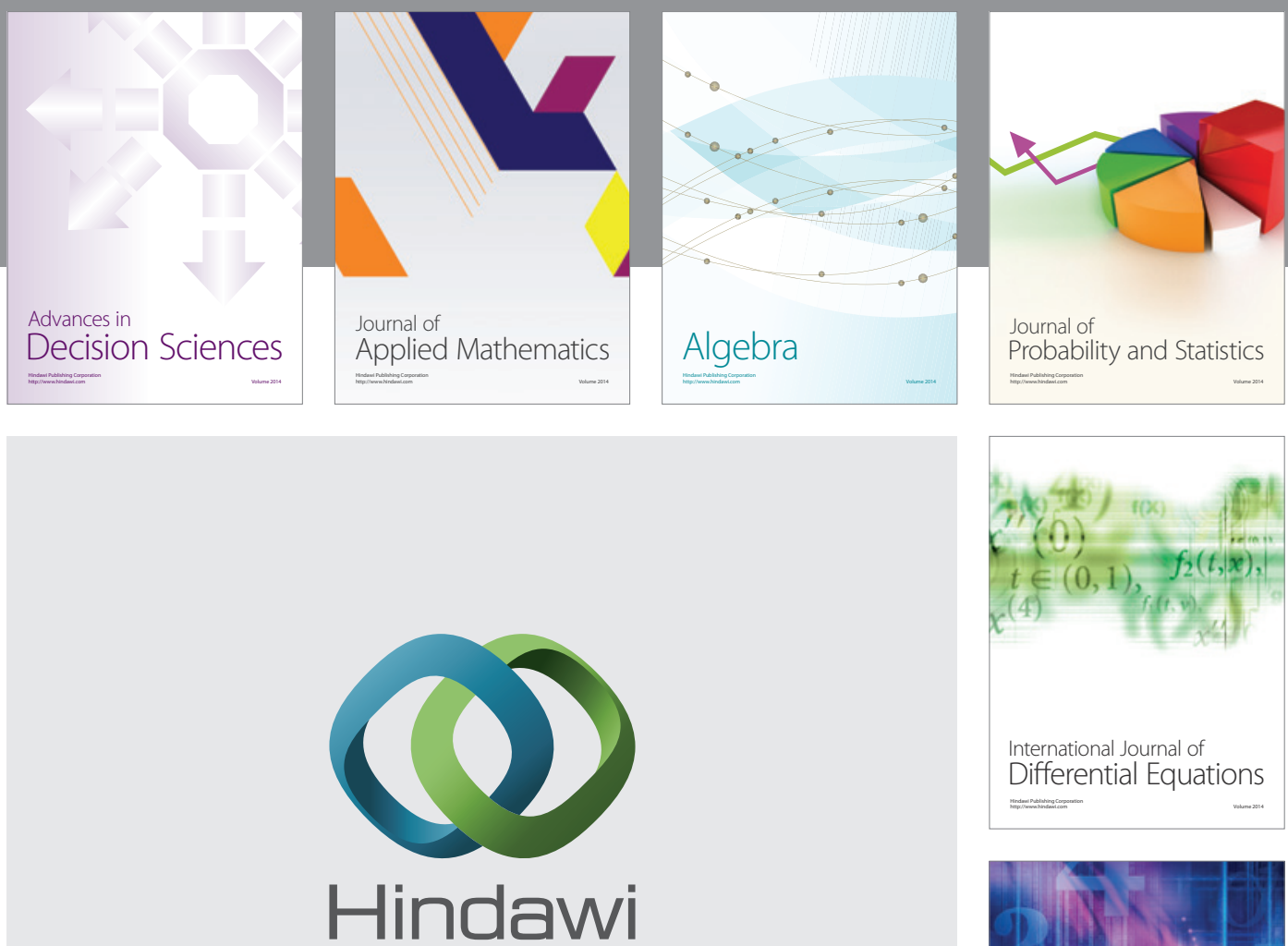

Submit your manuscripts at http://www.hindawi.com
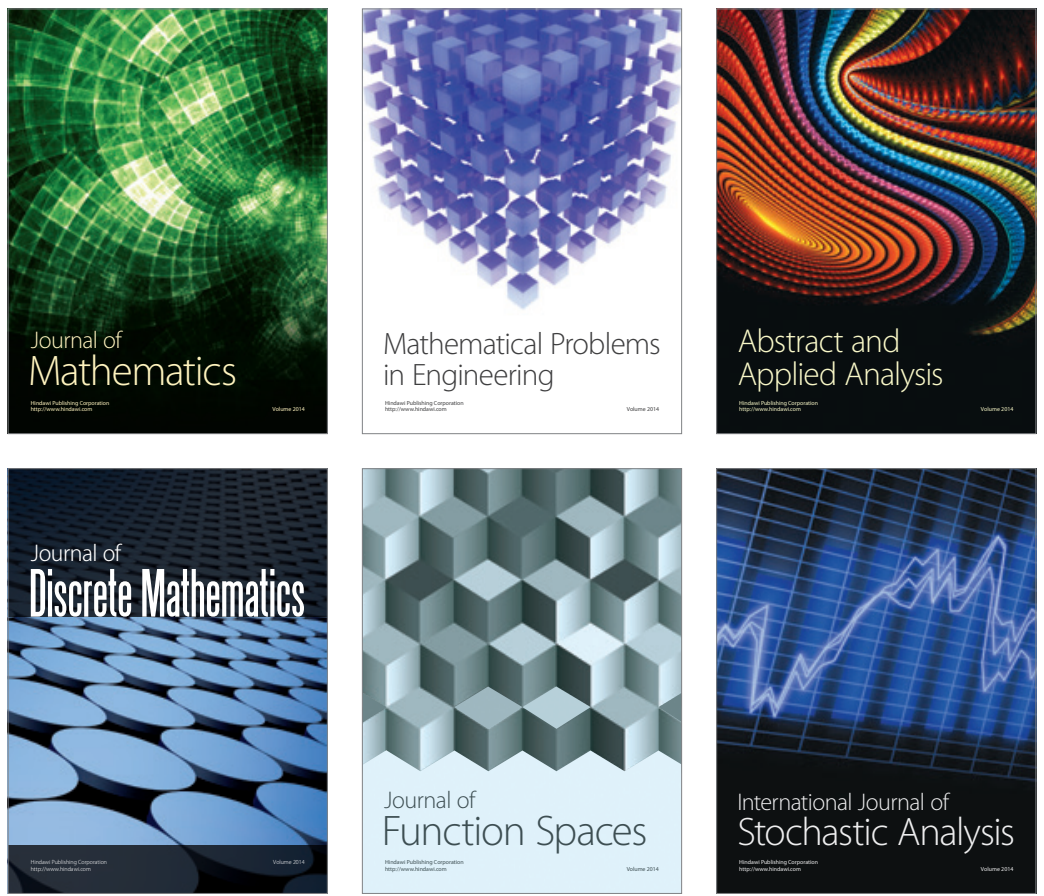

Journal of

Function Spaces

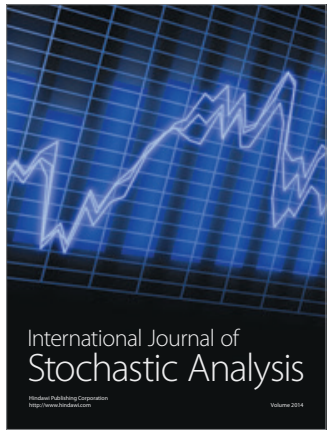

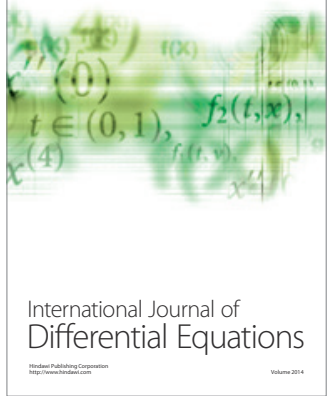
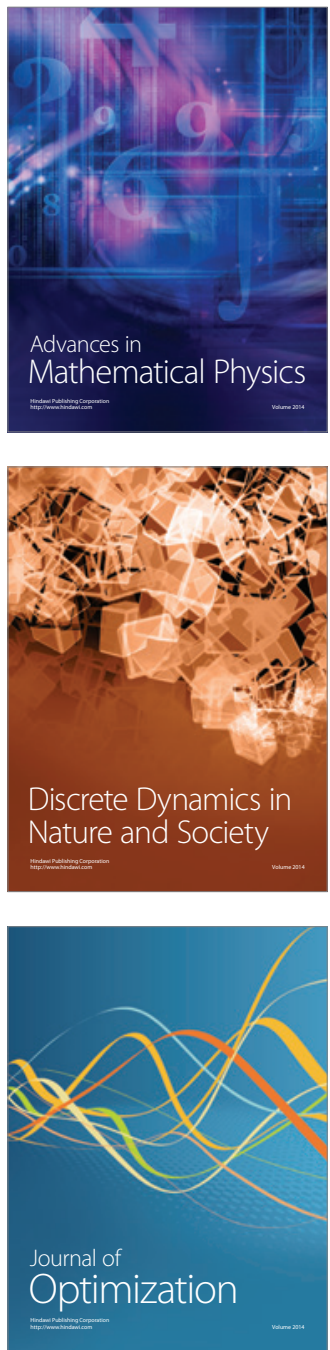